\title{
On the constraints violation in forward dynamics of multibody systems
}

\author{
Filipe Marques ${ }^{1}$, António P. Souto ${ }^{2}$ and Paulo Flores ${ }^{1, *}$ \\ ${ }^{1}$ Department of Mechanical Engineering, University of Minho \\ Campus de Azurém, 4804-533 Guimarães, Portugal \\ ${ }^{2}$ Department of Textile Engineering, University of Minho \\ Campus de Azurém, 4804-533 Guimarães, Portugal
}

\begin{abstract}
It is known that the dynamic equations of motion for constrained mechanical multibody systems are frequently formulated using the Newton-Euler's approach, which is augmented with the acceleration constraint equations. This formulation results in the establishment of a mixed set of partial differential and algebraic equations, which are solved in order to predict the dynamic behavior of general multibody systems. The classical resolution of the equations of motion is highly prone to constraints violation because the position and velocity constraint equations are not fulfilled. In this work, a general and comprehensive methodology to eliminate the constraints violation at the position and velocity levels is offered. The basic idea of the described approach is to add corrective terms to the position and velocity vectors with the intent to satisfy the corresponding kinematic constraint equations. These corrective terms are evaluated as function of the Moore-Penrose generalized inverse of the Jacobian matrix and of the kinematic constraint equations. The described methodology is embedded in the standard method to solve the equations of motion based on the technique of Lagrange multipliers. Finally, the effectiveness of the described methodology is demonstrated through the dynamic modeling and simulation of different planar and spatial multibody systems. The outcomes in terms of constraints violation at the position and velocity levels, conservation of the total energy and computational efficiency are analyzed and compared with those obtained with the standard Lagrange multipliers method, the Baumgarte stabilization method, the augmented Lagrangian formulation, the index-1 augmented Lagrangian and the coordinate partitioning method.
\end{abstract}

Keywords: Constraints violation, Baumgarte stabilization method, Penalty method, Augmented Lagrangian formulation, Index-1 Lagrangian formulation, Coordinate partitioning method, Mechanical energy, Computational efficiency, Forward dynamics, Multibody systems

\footnotetext{
*Corresponding author, Tel: + 351 253510220, Fax: +351 253516007, E-mail: pflores@dem.uminho.pt
} 


\section{Introduction}

Multibody dynamics deals with the study of the motion characteristics of many bodies whose interactions are modeled by forces and constraints. The problem of modeling and simulating constrained multibody systems has been widely studied over the last decades [1-9]. Recent review papers of interest on the formulation of multibody systems have been provided by Rahnejat [10], Udwadia [11] Eberhard and Schiehlen [12], Schiehlen [13] and Nikravesh [14]. The various formulations of dynamics of constrained multibody systems differ in the principle considered, type of coordinates adopted and the method selected for handling constraints in systems characterized by open and closed-loop topologies [15-22]. The solution of dynamic equations of motion for constrained multibody systems is often performed by using Lagrange multipliers technique, which leads to a set of differential and algebraic equations. Except for simple mechanical systems, the analytical solution of the equations of motion cannot be found, but in the most common cases require numerical resolution. In the standard Lagrange multipliers technique, the acceleration constraint equations are taken into account during the numerical resolution of the equations of motion and, therefore, there is no violation of the acceleration constraints. In sharp contrast, the position and velocity constraint equations are not utilized and, consequently, violation of the constraints at the position and velocity levels will occur due to the numerical integration errors. Thus, special procedures have to be implemented to eliminate or at least minimize the violation of the constraints [7, 23-30].

In a broad sense, the methods to handle the problem of constraints violation for dynamics of constrained multibody systems fall into three main categories: $(i)$ constraint stabilization approaches; (ii) coordinate partitioning methods and (iii) direct correct formulations [23]. The constraint stabilization approaches are probably the most popular due to their simplicity and easiness for computational implementation. However, their major drawback is the ambiguity in selecting the stabilization parameters, which ultimately can lead to failure simulations, even for systems that have valid solutions [31]. In turn, the coordinate partitioning methods have the great merit of allowing the rigorous resolution of the constraint equations at the position, velocity and acceleration levels. However, they can suffer from poor numerical efficiency when it is necessary to change the independent coordinate set [32]. The direct formulations, which include the projection methods, have physical meaning, computational efficiency, but they can exhibit some numerical instability [33]. There is no doubt that the constraint stabilization approaches and the direct correct formulations are the most popular and utilized methods to deal with the problem of constraints violation [7, 27-29, 34-44]. 
The Baumgarte stabilization method is the most widely utilized technique to overcome the limitations of the standard resolution of the dynamic equations of motion, which is borrowed from the feedback control theory [45]. The principle of this method is based on the addition of two terms to the acceleration constraint equations in order to stabilize the violations in the position and velocity constraint equations. This method has been extended to the problem of violation stabilization of holonomic systems formulated by using the canonical momenta approach [46]. The original Baumgarte method is very straightforward and easy to incorporate in any general multibody computational code [21]. In fact, this approach has been utilized successfully for many applications [47-59]. One of the problems associated with the Baumgarte stabilization method deals with the ambiguity in choosing feedback parameters, which eventually can seriously influence the dynamic equations of motion and get poor simulation results. In fact, as pointed out by Baumgarte [45], the selection of the stabilization parameters usually involves a trial and error procedure. The problem of selection of the stabilization parameters has been object of many research works over the last few decades. Ascher et al. [60] explained the difficulties of the parameter choice for Baumgarte stabilization and introduced improved constraint stabilization techniques. Chang and Nikravesh [61] presented an adaptive methodology for determining the optimum values of the stabilization parameters associated with the Baumgarte method. The effectiveness of this methodology was demonstrated through its application to two examples that illustrate the improvements in reducing the constraints violation. Bae and Yang [62] and Yoon and coworkers [63] developed different techniques to select the Baumgarte stabilization parameters. Lin and Hong [64] highlighted that the use to positive values for Baumgarte stabilization parameters is not sufficient to ensure convergence of the constraint equations at position and velocity levels. Lin and Huang [65] presented a method to select the stabilization parameters where the Runge-Kutta algorithm was employed. This work used the stability analysis method in digital control theory. More recently, Flores and his coworkers [31] presented a parametric study on the Baumgarte stabilization method. In this work the authors analyzed and discussed the influence of the stabilization parameters, integration method, time step and quality of the initial conditions on the dynamic response of planar constrained multibody systems.

Park and Chiou [66] developed a constraint stabilization approach similar to the Baumgarte method by using a penalty form of the constraint equations, which integrates two different sets of equations, one for the coordinates and another one for the Lagrange multipliers [67]. Ostermeyer [68], based on optimum control theory, improved the Baumgarte method. Yoon et al. [69] presented an effective method to deal with the energy constraint control in numerical 
simulation of constrained mechanical systems. The method is developed based on the geometric interpretation of the relation between constraints in the phase space. Several combinations of energy constraint control with either Baumgarte's constraint violation stabilization method or the new constraint violation stabilization using gradient feedback are also addressed. A new method for implementing constraint controls is developed by using the Euler method for integrating constraint control terms. Inspired on the seminal work of Baumgarte, Hong et al. [70] proposed an implicit constraint enforcement approach that is stable over large time steps and does not require problem dependent stabilization parameters. This technique utilizes the future time step to estimate the correct magnitude of the constraint forces, resulting in better stability over higher time steps. In addition, this formulation is physically conforming and minimizes the constraint drifts. In later work, Hong et al. [71] presented an implicit holonomic and non-holonomic constraint enforcement method for rigid body dynamics that ensures numerical stability without requiring ad-hoc stabilization parameters. This technique provides the same asymptotic computational cost as the Baumgarte method. Weijia and co-authors [72], also based on the Baumgarte method, presented an automatic formulation for the constraints violation stabilization of the numerical solution of the equations of motion in the context of dynamics of multibody systems. They use the Taylor expansion series to determine a relation between the stabilization parameters and the integration time step. Cline and Pai [73] proposed a post-stabilization approach for rigid multibody systems simulation. This approach compensates the error of integration each time step to correct the constraint error. However, the post-stabilization requires a linear system solution to find the error correction and it does not preserve the correct dynamic motion of objects when it reduces constraint drifts. Alternative techniques to deal with the problem of the constraint violation stabilization have been proposed by many researchers [7478]. A detailed and complete review on the main approaches for constraint enforcement in the context of multibody dynamics has been performed by Bauchau and Laulusa [29] and Vlasenko and Kasper [41].

In the coordinate partitioning method, the generalized coordinates are partitioned into independent and dependent sets [79], being the numerical integration carried out for independent generalized coordinates. Then, the constraint equations are solved for dependent generalized coordinates. The advantage of this method is that it satisfies all the constraints to the level of precision specified and maintains good error control. However, it can suffer from poor numerical efficiency when it is necessary to change the independent coordinate set. Nikravesh and Haug [80] proposed a comprehensive method based on the generalized coordinate partitioning for analysis of multibody systems with holonomic and non-holonomic constraints. In this work, a 
Gaussian elimination scheme with full pivoting is utilized to decompose the constraint Jacobian matrix and identifies independent coordinates. Haug and Yen [81] also investigated on the coordinate partitioning approach for numerical integration of differential-algebraic equations under the framework of multibody dynamics. The accuracy of this formulation has been demonstrated, both from theoretical and numerical points of view. Fisette and Vaneghem [33], based on the coordinate partitioning method, used the LU factorization of constraints Jacobian matrix to identify the dependent and independent coordinates. This aspect is of paramount importance since during the integration process, numerical problems may arise due to inadequate choice of the independent coordinates that lead to poorly conditioned matrices. This problem was also considered by Arabyan and $\mathrm{Wu}$ [82] to study multibody mechanical systems with both holonomic and non-holonomic constraints. Neto and Ambrósio [32] also utilized the coordinate partitioning method to handle the constraints violation correction for the integration of differential algebraic equations in the presence of redundant constraints. It must be highlighted that the coordinate partitioning methods are effective and very useful [83-85] and a good number of investigations haven been carried out on the establishment of the sets of independent and dependent coordinates. In fact, over the last few decades, several approaches that allows for the selection of the independent set of coordinates have been proposed [86-91]. Recently, Carpinelli et al. [92] studied the problem of the accuracy and efficiency of the implicit Euler approach when switching from dependent to independent coordinates. For this, an automatic procedure is implemented.

In the direct correct formulations, the violation of constraint equations are eliminated during the numerical resolution of the DAE equations of motion [29]. Over the last years, several techniques have been developed to eliminate the constraints violation, namely those by Bayo et al. [74] Lubich [93], Andrzejewski and Bock [94], Yu and Chen [95] and Blajer [27]. Eich [96] presented a coordinate projection method that allows for the control of the constraints violation in mechanical multibody systems with algebraic constraints. Yoon et al. [97] presented a direct correction formulation to eliminate the violation of the constraints in numerical simulation of constrained multibody systems. This method corrects the values of the state variables directly so that they can fit the constraint equations well. However, this method is formulated at the positions level only. Blajer [98] considered the projection method to obtain the dynamic equations of motion for constrained multibody systems in the form of ordinary differential equations. Then, a standard solver was used to integrate the resulting system. In a later work Blajer [99] presented a unified geometric formulation for constrained multibody systems. Blajer highlighted that the negligence of the inertial properties of the systems can result in physical 
inconsistency. A method that allows for the elimination of the constraints violation has been proposed by Aghili and Piedboeuf [100], which is based on the concept of pseudo-inverse of the constraint matrix. This formulation is able to solve the problem associated with redundant constraints and singularities in mechanical multibody systems. Tseng et al. [101] used the Maggi's equations with perturbed iteration to develop an efficient approach to numerically solve the dynamic equations of motion of constrained multibody systems. This approach, named integrate-and-collaborate paradigm, has the goal to execute coupled system simulation without sacrificing the integrity of subsystem modeling and solutions, and to maintain the effectiveness of the overall results. Nikravesh [102] proposed a direct method to obtain appropriate the initial coordinates and velocities, where the basic idea is to correct the values of the state variables before the numerical solution of the dynamic equations of motion take place. Recently, Zhang et al. [103] presented a physically consistent methodology to suppress the violation of the constraint equations of three-dimensional multibody systems, in which the mass matrix is singular. For this purpose, the constrained and weighted least-squares-based geometrical projection method is considered in the resolution of the equations of motion, and the explicit correction formulation is formulated by the block matrix inversion procedure. The effectiveness of this methodology is demonstrated trough several benchmark examples of application, where the constraints at the position level are precisely ensured with a few number of iterations, while the constraints at the velocity level are guaranteed with a single step procedure.

Alternative methodologies to handle the elimination of the constraints violation have been addressed by other researchers, being the interested reader referred to the following references [35, 75, 76, 83, 84, 104-106]. For instance, Cuadrado and his coworkers [35] presented a comparative analysis for four methodologies to handle constrained multibody systems, namely the augmented Lagrangian formulation with projections in index-1 and index-3, a modified state-space formulation based on the projection matrices and a fully-recursive formulation. Four different benchmark problems have been considered to effectively assess the influence of the approach utilized in terms of computational efficiency, accuracy and performance. Terze et al. $[76,83,84]$ proposed an integration method for dynamic simulation of constrained multibody systems with no constraint violation. In a first stage, the set of independent variables are identified and selected, and then the displacement constraint violations were eliminated in an iterative process. In a second stage, considering the constraints equations at the velocity level eliminated the velocity constraint violations. In turn, Yu and Chen [95] presented a direct violation correction approach both at the position and velocity levels. The effectiveness of the approach was validated and compared with standard formulation and 
Baumgarte method applied to a simple planar four bar mechanism. Bayo and Ledesma [104] combined the well-established augmented Lagrangian formulation together with a mass orthogonal projection technique for constrained multibody systems. This particular approach has been successfully employed by many researchers, due to its computational robustness, accuracy and efficiency. In fact, the utilization of the mass orthogonal projection technique is quite effective and minimizes the constraints violation at the machine accuracy level. Orden and coauthors $[105,106]$ investigated on the energy balance of a velocity projection providing an alternative interpretation of its effect on the stability and a practical criterion for the massorthogonal projection matrix selection. More recently, Terze and coworkers [107, 108] has investigated on the Lie-group integration method for constrained multibody systems in state space. The proposed approach avoids the kinematical differential equations because the formulation integration algorithm is developed on the system manifold via MBS element's angular velocities and rotational matrices. This is of paramount importance since they can eliminate the problems associated with singularities. With the purpose of eliminating the numerical constraint violations at both the positions and velocity levels during the integration scheme, a projection method based on constrained least-square minimization algorithm was presented. The easiness and effectiveness of the proposed approach was demonstrated throughout the simulation of two numerical examples of application, namely the heavy top dynamics and the satellite with mounted 5-DOF manipulator. The approach seems to be especial useful for discrete mechanical systems that incorporate general kinematical constraints and for systems that describes large 3D rotations [107].

The main emphasis of this work is on the elimination of the constraints violation during the dynamic analysis of constrained multibody systems. Body coordinates formulation is used to describe the system components and the kinematic joints. The equations governing the dynamic behavior of the general mechanical systems incorporate corrective terms that are added to the position and velocity vectors in order to satisfy the corresponding constraint equations. These corrective terms are expressed in terms of the Jacobian matrix and kinematic constraint equations. Moreover, the corrective terms are added and considered during the numerical resolution of the dynamic equations of motion. In the sequel of this process, the standard method based on the Lagrange multipliers technique, the Baumgarte stabilization method, the coordinating partitioning method, the penalty approach and the augmented Lagrangian formulation have been revisited. Results for several planar and spatial multibody mechanical systems are presented and utilized to discuss the assumptions and procedures adopted throughout this work. 


\section{Equations of motion for constrained multibody systems}

In a simple manner, a mechanical multibody system embraces two main features, namely $(i)$ mechanical components that describe large translational and rotational motions and (ii) kinematic joints that impose some constraints on the relative motion of the bodies [2-4]. Figure 1 depicts a generic configuration of a spatial multibody system that encompasses a collection of rigid and flexible bodies interconnected by kinematic joints and acted upon by force elements. The forces applied over the multibody system components can be the result of springs, dampers, actuators or external forces. External applied forces of different nature and different levels of complexity can act on a multibody system with the purpose to simulate the interaction among the system components and between these and the surrounding environment [109, 110].

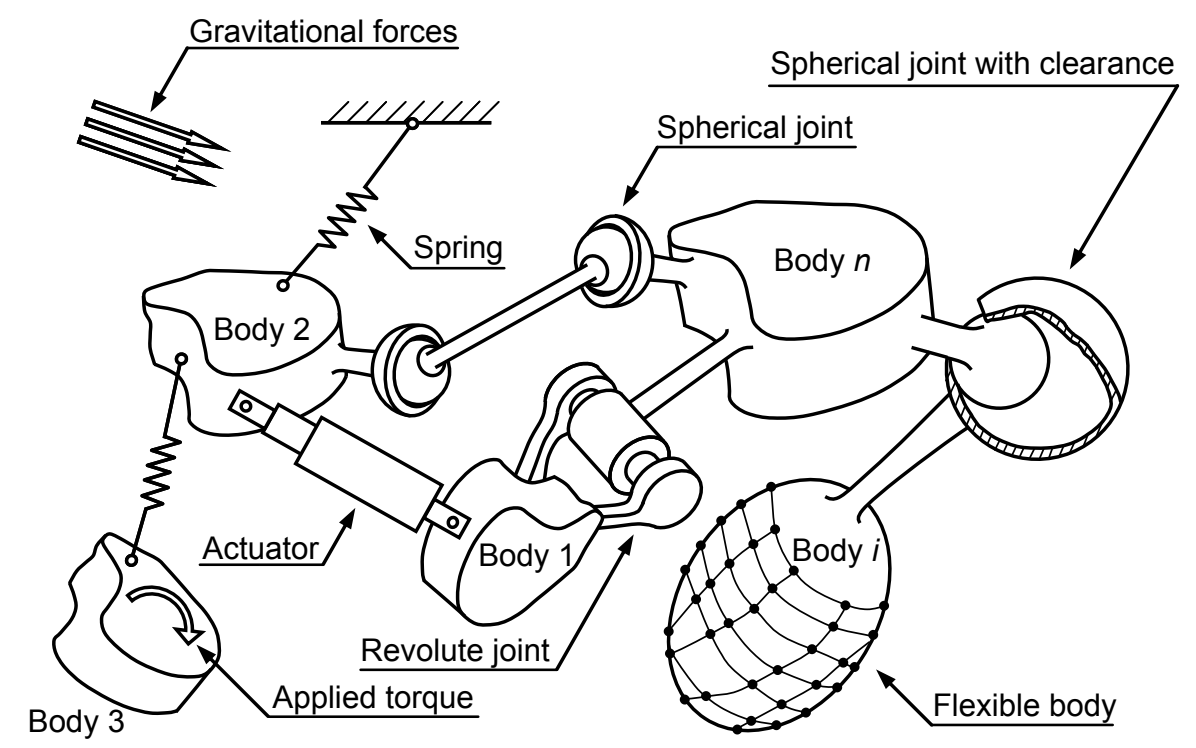

Fig. 1 Generic representation of a mechanical multibody system with its most significant components: bodies, joints and forces elements.

In order to be able to analyze the dynamic response of constrained mechanical multibody systems, it is first necessary to formulate the equations of motion that govern the behavior of multibody systems. For this purpose, let the configuration of a constrained mechanical multibody system be described by $n$ generalized coordinates, then a set of $m$ algebraic kinematic independent scleronomic constraints $\boldsymbol{\Phi}$ can be written in a compact form as [2]

$$
\boldsymbol{\Phi} \equiv \boldsymbol{\Phi}(\mathbf{q})=\mathbf{0}
$$

where $\mathbf{q}$ is the vector of generalized coordinates. The number of generalized coordinates, $n$, and the number of kinematic constraints, $m$, must be adequately selected, bearing in mind the correct system's description and system's degrees of freedom [21]. In the most general case, the 
constraint equation (1) encompasses the total set of holonomic and non-holonomic restrictions to which a generic multibody system can be subjected [11]. The velocities and accelerations of the system components are evaluated by using the velocity and acceleration constraint equations. Thus, the first time derivative of Eq. (1) provides the velocity constraint equations as

$$
\dot{\boldsymbol{\Phi}} \equiv \mathbf{D v}=\mathbf{0}
$$

where $\mathbf{D}$ denotes the system Jacobian matrix and $\mathbf{v}$ is the vector of generalized velocities.

A second differentiation of Eq. (1) with respect to time leads to acceleration constraint equations as

$$
\ddot{\boldsymbol{\Phi}} \equiv \mathbf{D} \dot{\mathbf{v}}+\dot{\mathbf{D}} \mathbf{v}=\mathbf{0}
$$

in which $\dot{\mathbf{v}}$ is the generalized accelerations and the term $-\dot{\mathbf{D}} \mathbf{v}$ is referred to as the right-hand side of the acceleration equations. By introducing $\gamma=-\dot{\mathbf{D}} \mathbf{v}$, Eq. (3) can be rewritten as

$$
\mathbf{D} \dot{\mathbf{v}}=\gamma
$$

For a multibody system of constrained bodies, the Newton-Euler equations of motion are written as $[2,4]$

$$
\mathbf{M} \dot{\mathbf{v}}=\mathbf{g}+\mathbf{g}^{(c)}
$$

where $\mathbf{M}$ is the global mass matrix, $\mathbf{g}$ is the vector of generalized forces and $\mathbf{g}^{(c)}$ denotes the vector of reaction forces that can be expressed in terms of the constraints Jacobian matrix and Lagrange multipliers, $\lambda$, as

$$
\mathbf{g}^{(c)}=\mathbf{D}^{T} \boldsymbol{\lambda}
$$

Finally, the translational and rotational equations of motion for a constrained multibody system can be expressed in its general form as

$$
\mathbf{M} \dot{\mathbf{v}}-\mathbf{D}^{T} \boldsymbol{\lambda}=\mathbf{g}
$$

In dynamic analysis, a unique solution is obtained when the algebraic constraint equations at the acceleration level are considered simultaneously with the differential equations of motion together with a set of appropriate initial conditions [4]. Therefore, Eq. (4) is appended to Eq. (7), yielding a system of differential and algebraic equations written as

$$
\left[\begin{array}{cc}
\mathbf{M} & -\mathbf{D}^{T} \\
\mathbf{D} & \mathbf{0}
\end{array}\right]\left\{\begin{array}{c}
\dot{\mathbf{v}} \\
\boldsymbol{\lambda}
\end{array}\right\}=\left\{\begin{array}{l}
\mathbf{g} \\
\boldsymbol{\gamma}
\end{array}\right\}
$$

This linear system of equations can be solved by applying any method suitable for the resolution of linear algebraic equations. The existence of null elements in the main diagonal of 
the leading matrix and the possibility of ill-conditioned matrices suggest that methods using partial or full pivoting are preferred. The dynamic equations of motion can also be solved analytically. For this purpose, Eq. (7) is rearranged to put the accelerations vector in evidence, yielding

$$
\dot{\mathbf{v}}=\mathbf{M}^{-1}\left(\mathbf{g}+\mathbf{D}^{T} \lambda\right)
$$

In this process, it is assumed that the inverse of the mass matrix $\mathbf{M}$ exists. It must be emphasized that a unique solution of Eq. (8) is guaranteed when the mass matrix is positive definite and the Jacobian matrix has a maximum rank [4]. This particular issue has been analyzed in detail in the seminal work by Jalón and Gutiérrez-López [90]. Introducing Eq. (9) into Eq. (4) and after basic mathematical manipulation results in

$$
\lambda=\left[\mathbf{D M}^{-1} \mathbf{D}^{T}\right]^{-1}\left(\boldsymbol{\gamma}-\mathbf{D} \mathbf{M}^{-1} \mathbf{g}\right)
$$

Substituting now Eq. (10) into Eq. (9) yields

$$
\dot{\mathbf{v}}=\mathbf{M}^{-1} \mathbf{g}+\mathbf{M}^{-1} \mathbf{D}^{T}\left\{\left[\mathbf{D} \mathbf{M}^{-1} \mathbf{D}^{T}\right]^{-1}\left(\boldsymbol{\gamma}-\mathbf{D} \mathbf{M}^{-1} \mathbf{g}\right)\right\}
$$

Thus, Eq. (11) is solved for $\dot{\mathbf{v}}$ then, the velocities and positions can be obtained by numerical integration. This procedure must be repeated until the final time of analysis is reached. This manner to solve the dynamic equations of motion is commonly referred to as the standard Lagrange multipliers method [2].

At this stage, it must be highlighted that some numerical difficulties can arise when solving the dynamic equations of motion. As already stated, in general, it is assumed that the mass matrix is always invertible. However, it has been demonstrated in many research studies that the mass matrix can be singular, namely when more than six coordinates are considered to define the pose of a rigid body [39, 41, 90, 111, 112]. Another problem can appear when a body in the system under analysis has extremely small inertia [39, 90, 111]. A third problem that often can take place is associated with redundant constraints. Additional difficulties that can occur in the resolution of the equations of motion are related to systems with changing topologies [22] and units [113]. Within the spirit of the present study, these issues are not object of investigation, being the interested reader referred to the following references $[4,22,23,32,42,43,76,90,101$, 105, 111-118]. 
The system of the equations of motion (8) or (11) does not use explicitly the position and velocity equations associated with the kinematic constraints, that is, Eqs. (1) and (2). In other words, after the numerical resolution of the Eq. (8), both constraints at the position and velocity levels are not satisfied. Consequently, for moderate or long simulations, the original constraint equations start to be violated due to the integration process and/or to inaccurate initial conditions. Therefore, methods able to eliminate errors in the position and velocity equations or, at least, to keep such errors under control, must be adopted. In order to keep the constraint violations under control, the Baumgarte stabilization method is considered here [45]. This method allows constraints to be slightly violated before corrective actions can take place, in order to force the violation to vanish. The objective of Baumgarte method is to replace the differential equation (3) by the following expression

$$
\ddot{\boldsymbol{\Phi}}+2 \alpha \dot{\boldsymbol{\Phi}}+\beta^{2} \boldsymbol{\Phi}=\mathbf{0}
$$

Equation (12) is a differential equation for a closed-loop system in terms of kinematic constraint equations, in which the terms $2 \alpha \dot{\Phi}$ and $\beta^{2} \boldsymbol{\Phi}$ play the role of control terms. The principle of the method is based on the damping of acceleration of constraints violation by feeding back the position and velocity of constraints violation. Figure 2 illustrates open and closed-loop control systems. It is known that in the open-loop systems $\boldsymbol{\Phi}$ and $\dot{\boldsymbol{\Phi}}$ do not converge to zero if any perturbation occurs and, therefore, the system is unstable. Thus, using the Baumgarte approach, the equations of motion for a system subjected to kinematic constraints can be stated in the following form

$$
\left[\begin{array}{cc}
\mathbf{M} & -\mathbf{D}^{T} \\
\mathbf{D} & \mathbf{0}
\end{array}\right]\left\{\begin{array}{c}
\dot{\mathbf{v}} \\
\boldsymbol{\lambda}
\end{array}\right\}=\left\{\begin{array}{c}
\mathbf{g} \\
\gamma-2 \alpha \dot{\boldsymbol{\Phi}}-\boldsymbol{\beta}^{2} \boldsymbol{\Phi}
\end{array}\right\}
$$

If the gain parameters, $\alpha$ and $\beta$, are chosen as positive constants, in general systems, the stability of the general solution of Eq. (13) is guaranteed. Baumgarte [45] highlighted that the suitable choice of the parameters $\alpha$ and $\beta$ can be performed by numerical experiments. Hence, the Baumgarte method has some ambiguity in determining optimal feedback gains. The improper choice of these parameters can lead to unacceptable results in the dynamic analysis of the multibody systems. Over the last decades, a good number of works have been published on the selection of the gain parameters and their influence on the systems' response $[31,47,51,53,55$ $58,60,63,70,71,119]$. 


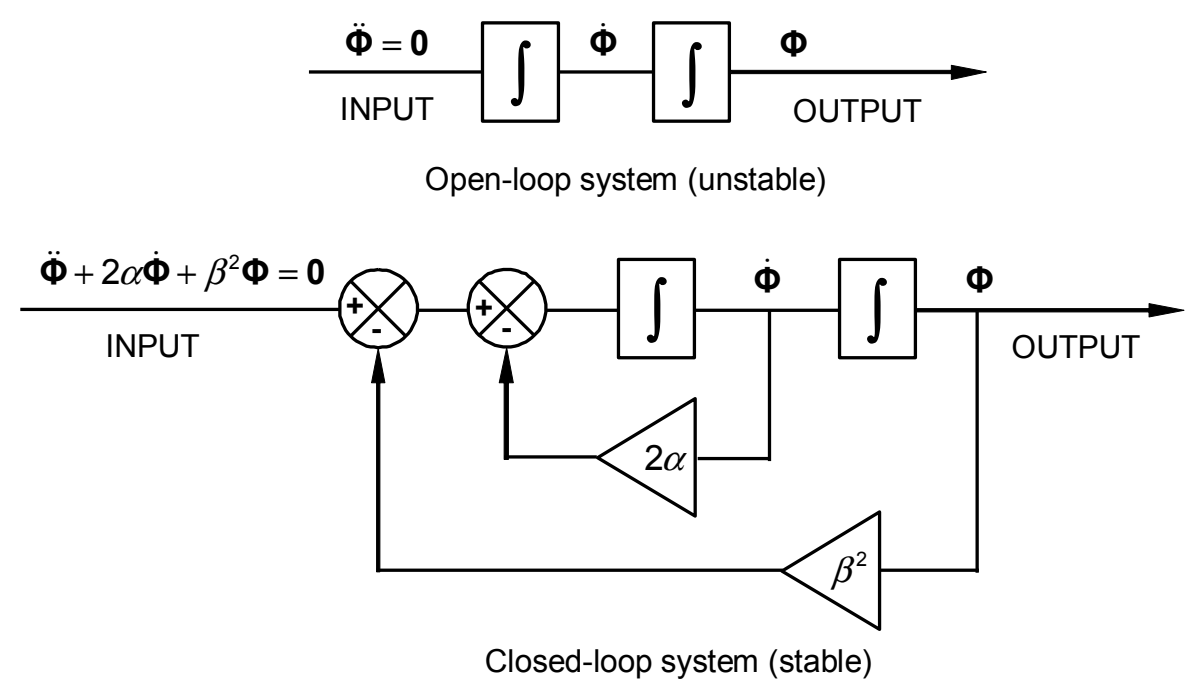

Fig. 2 Open-loop and closed-loop control systems.

The coordinate partitioning method requires that the system coordinates to be partitioned into two sets, independent and dependent coordinates [79]

$$
\mathbf{q} \Rightarrow\left\{\begin{array}{c}
\mathbf{q}^{(i)} \\
\mathbf{q}^{(d)}
\end{array}\right\}
$$

where superscripts $(i)$ and $(d)$ denote independent and dependent, respectively. The same partitioning is also applied to the array of velocities and accelerations. The integration process of the equations of motion is revised to integrate only for the independent variables, that is

$$
\left\{\begin{array}{c}
\mathbf{v}^{(i)} \\
\dot{\mathbf{v}}^{(i)}
\end{array}\right\} \rightarrow \int \rightarrow\left\{\begin{array}{c}
\mathbf{q}^{(i)} \\
\mathbf{v}^{(i)}
\end{array}\right\}
$$

Dependent coordinates are determined by substituting $\mathbf{q}^{(i)}$ in Eq. (1) and solving the nonlinear constraints through an iterative scheme such as Newton-Raphson [2]. Dependent velocities are obtained by substituting $\mathbf{v}^{(i)}$ in Eq. (2) and solving the resultant set of linear equations. Then Eq. (8) is solved to determine the entire set of accelerations, and the integration process of Eq. (15) is repeated for the next time step.

The coordinate partitioning method eliminates the constraint violations completely since all the constraints, at the coordinate, velocity, and acceleration levels are solved for. The accuracy of the coordinate partitioning method depends on the selection of the set of independent coordinates. Furthermore, it should be noted that the number of independent variables should be equal to the number of degrees-of-freedom of the system. The identification of the dependent and independent variables can be done automatically by using, for instance, a matrix 
factorization technique, such as Gaussian elimination with full pivoting [32]. One drawback of this method is that the choice of the independent variables may change during a simulation, mostly due to large rotations of bodies. Although switching from one set of independent variable to another during a simulation is possible, the associated computational overhead can be costly. Furthermore, the computational effort associated with the Newton-Raphson process can, in some cases, be considered as another drawback. A good number of researchers have been utilized the coordinate partitioning method in the field of multibody dynamics $[23,32,33,79-82,86,87$, 120-122]. It has been stated by some authors that the coordinate partitioning method is not very efficient from numerical point of view due to the necessity to solve the nonlinear constraint equations and also due to the need to change the independent coordinates. However, the coordinate partitioning method can be very interesting and efficient when combined with the Maggi's formulation, as it has been shown in the literature [28, 101, 123, 124].

The penalty method presented by Jalón and Bayo [7] constitutes an alternative way to solve the dynamic equations of motion. In this method, the equations of motion are modeled as a linear second-order differential equation that can be written in the form

$$
m_{c} \ddot{\boldsymbol{\Phi}}+d_{c} \dot{\boldsymbol{\Phi}}+k_{c} \boldsymbol{\Phi}=\mathbf{0}
$$

Introducing Eq. (3) into Eq. (16) yields

$$
m_{c}(\mathbf{D} \dot{\mathbf{v}}+\dot{\mathbf{D}} \mathbf{v})+d_{c} \dot{\boldsymbol{\Phi}}+k_{c} \boldsymbol{\Phi}=\mathbf{0}
$$

Pre-multiplying Eq. (17) by the transpose of Jacobian matrix, $\mathbf{D}^{T}$, and after mathematical treatment, results in

$$
m_{c} \mathbf{D}^{T} \mathbf{D} \dot{\mathbf{v}}=-\mathbf{D}^{T}\left(m_{c} \dot{\mathbf{D}} \mathbf{v}+d_{c} \dot{\boldsymbol{\Phi}}+k_{c} \boldsymbol{\Phi}\right)
$$

Let consider now the Newton-Euler equations of motion for a system of unconstrained system and written here as [2]

$$
\mathbf{M} \dot{\mathbf{v}}=\mathbf{g}
$$

Adding Eqs. (18) and (19) yields

$$
\mathbf{M} \dot{\mathbf{v}}+m_{c} \mathbf{D}^{T} \mathbf{D} \dot{\mathbf{v}}=\mathbf{g}-\mathbf{D}^{T}\left(-m_{c} \gamma+d_{c} \dot{\boldsymbol{\Phi}}+k_{c} \boldsymbol{\Phi}\right)
$$

in which Eq. (4) has been employed. Finally, Eq. (20) can be rewritten in the following form

$$
\left(\mathbf{M}+\alpha \mathbf{D}^{T} \mathbf{D}\right) \dot{\mathbf{v}}=\mathbf{g}-\alpha \mathbf{D}^{T}\left(-\gamma+2 \mu \omega \dot{\Phi}+\omega^{2} \boldsymbol{\Phi}\right)
$$

where

$$
\alpha=m_{c}, \quad d_{c}=2 \mu \omega m_{c} \quad \text { and } \quad k_{c}=\omega^{2} m_{c}
$$


Equation (21) can be solved for $\dot{\mathbf{v}}$. This penalty method gives good results if $\alpha$ tends to infinity. Typical values of $\alpha, \omega$ and $\mu$ are $1 \times 10^{7}, 10$ and 1 , respectively [7, 125]. It should be noted that with this penalty method, multibody systems with redundant constraints or kinematic singular configurations can be solved. In fact, the penalty method has been utilized to evaluate the constraint forces in multibody systems with redundant constraints $[111,117,118]$. However, this approach cannot handle the problem of solving indetermination of the Lagrange multipliers, as it was analyzed by Jalón and Gutiérrez-López [90].

The augmented Lagrangian formulation, which can be seen as an evolution of the penalty method, is a methodology that penalizes the constraints violation, much in the same form as the Baumgarte stabilization method. This is an iterative procedure that presents advantages relative to other methods because it involves the solution of a smaller set of equations, handles redundant constraints and still delivers accurate results in the vicinity of singular configurations [7, 104]. Let index $i$ denote the $i$-th iteration. Thus, based on the work by Bayo and Ledesma [104], the evaluation of the system accelerations in a given time step starts as

$$
\mathbf{M} \dot{\mathbf{v}}_{i}=\mathbf{g}, \quad(i=0)
$$

The iterative process to evaluate the system accelerations proceeds with the evaluation of

$$
\left(\mathbf{M}+\alpha \mathbf{D}^{T} \mathbf{D}\right) \dot{\mathbf{v}}_{i+1}=\mathbf{M} \dot{\mathbf{v}}_{i}-\alpha \mathbf{D}^{T}\left(-\gamma+2 \mu \omega \dot{\Phi}+\omega^{2} \boldsymbol{\Phi}\right)
$$

This iterative process continues until

$$
\left\|\dot{\mathbf{v}}_{i+1}-\dot{\mathbf{v}}_{i}\right\|=\varepsilon
$$

where $\varepsilon$ is a specified tolerance [104].

The augmented Lagrangian method involves the solution of a system of equations with a dimension equal to the number of coordinates of the multibody system. Though mass matrix $\mathbf{M}$ is generally positive semi-definite the leading matrix of Eq. (24) $\mathbf{M}+\alpha \mathbf{D}^{T} \mathbf{D}$ is always positive definite [7, 29]. Bayo and coworkers [126] extended and simplified the original augmented Lagrangian formulation and demonstrated that the penalty terms associated with the velocity constraints are needed to avoid the high frequency oscillations that can appear during the numerical resolution of the equations of motion. It has been shown by many researchers that the implementation of the augmented Lagrangian formulation is quite effective, efficient and robust when performing forward dynamic simulations. Finally, it must be stated that the selection of the penalty factors must avoid the numerical ill problems associated with flexibility of bodies, compliance of kinematic joints and manufacturing and assemble errors [90, 101, 104, 127]. 
Alternative formulations based on projections onto the constraints manifolds to fulfill the constraint equations have been proposed over the last decades, such as those by Bayo and Ledesma [104]. In particular, the index-1 augmented Lagrangian method with mass-orthogonal projections, which is based on the augmented Lagrangian formulation, utilizes the projections of positions and velocities to completely satisfy the constraints equations. In contrast to the other approaches, the index-1 augmented Lagrangian method is not applied during the resolution of the dynamic equations of motion. Thus, the generalized positions and velocities of the system, $\mathbf{q}_{0}$ and $\mathbf{v}_{0}$, respectively, are calculated using any integration scheme, which does not ensure the fulfillment of the constraints equations at position and velocity levels.

A mass-orthogonal projection of the solution is performed in order to obtain a corrected set of positions, which enforces the application of the following iterative procedure according to

$$
\left(\mathbf{M}+\alpha \mathbf{D}^{T} \mathbf{D}\right) \Delta \mathbf{q}_{i+1}=-\mathbf{M}\left(\mathbf{q}_{i}-\mathbf{q}_{0}\right)-\mathbf{D}^{T} \boldsymbol{\lambda}_{i}
$$

with

$$
\begin{aligned}
& \mathbf{q}_{i+1}=\mathbf{q}_{i}+\Delta \mathbf{q}_{i+1} \\
& \lambda_{i+1}=\lambda_{i}+\alpha \boldsymbol{\Phi}_{i+1}
\end{aligned}
$$

Similarly to Eq. (25), this iterative procedure involving Eqs. (26)-(28) is applied until the following condition is verified

$$
\left\|\Delta \mathbf{q}_{i+1}\right\|=\varepsilon
$$

It is clear that when the initial approximation $\mathbf{q}_{0}$ is close to the corrected set of coordinates, the modified Newton-Raphson method can be employed to improve the efficiency of this method.

A similar mass-orthogonal projection is performed at the velocity level. The inaccurate set of velocities obtained from numeric integration, $\mathbf{v}_{0}$, is updated solving the following system [104]

$$
\begin{gathered}
\left(\mathbf{M}+\alpha \mathbf{D}^{T} \mathbf{D}\right) \mathbf{v}_{i+1}=\mathbf{M} \mathbf{v}_{i}-\alpha \mathbf{D}^{T} \mathbf{v} \quad \text { (for rheonomic constraints) } \\
\left(\mathbf{M}+\alpha \mathbf{D}^{T} \mathbf{D}\right) \mathbf{v}_{i+1}=\mathbf{M} \mathbf{v}_{i} \quad \text { (for scleronomic constraints ) }
\end{gathered}
$$

It can be observed that the leading matrix $\mathbf{M}+\alpha \mathbf{D}^{T} \mathbf{D}$ depends only on the generalized positions of the system, therefore, it does not need to be updated in each iteration. The interested reader in formulations based on projections onto constraints manifolds is referred to the recent work by Cuadrado et al. [128], Dopico et al. [129.] and González et al. [130, 131]. 


\section{Direct correction approach to eliminate the constraints violation}

The main purpose of this section is to present a general and comprehensive direct correction approach to deal with the elimination of the constraints violation at both position and velocity levels. For this, let consider that during the numerical resolution of the dynamic equations of motion, the vector of generalized coordinates needs to be corrected due to the constraints violation. Thus, the corrected positions can be expressed in the form

$$
\mathbf{q}^{c}=\mathbf{q}^{u}+\delta \mathbf{q}
$$

where $\mathbf{q}^{u}$ denotes the uncorrected positions and $\delta \mathbf{q}$ represents the set of corrections that

eliminates the constraints violation. This means that the corrective term has to be added to vector $\mathbf{q}^{u}$ in order to ensure that the constraint equations (1) are satisfied, that is

$$
\Phi\left(\mathbf{q}^{c}\right)=\boldsymbol{\Phi}\left(\mathbf{q}^{u}\right)+\delta \Phi=\mathbf{0}
$$

The term $\delta \Phi$ in Eq. (32) can be understood as the variation of the constraint equations and can be expressed as [132]

$$
\delta \Phi=\frac{\partial \Phi}{\partial \mathbf{q}_{1}} \delta \mathbf{q}_{1}+\frac{\partial \Phi}{\partial \mathbf{q}_{2}} \delta \mathbf{q}_{2}+\ldots+\frac{\partial \Phi}{\partial \mathbf{q}_{n}} \delta \mathbf{q}_{n}=\mathbf{D} \delta \mathbf{q}
$$

Combining now Eqs. (32) and (33) yields

$$
\Phi\left(\mathbf{q}^{u}\right)+\mathbf{D} \delta \mathbf{q}=\mathbf{0}
$$

which ultimately leads to

$$
\delta \mathbf{q}=-\mathbf{D}^{-1} \boldsymbol{\Phi}\left(\mathbf{q}^{u}\right)
$$

In general, the Jacobian matrix, $\mathbf{D}$, is not square, therefore, $\mathbf{D}^{-1}$ does not exist. However, the concept of the Moore-Penrose inverse matrix, $\mathbf{D}^{+}$, can be employed as [133-135]

$$
\mathbf{D}^{+}=\mathbf{D}^{T}\left(\mathbf{D} \mathbf{D}^{T}\right)^{-1}
$$

such that

$$
\begin{aligned}
\mathbf{D D}^{+} \mathbf{D} & =\mathbf{D} \\
\mathbf{D}^{+} \mathbf{D D}^{+} & =\mathbf{D}^{+}
\end{aligned}
$$

and both $\mathbf{D}^{+} \mathbf{D}$ and $\mathbf{D D} \mathbf{D}^{+}$are symmetric matrices. Consequently, it is possible to establish the following mathematical relation [82],

$$
\mathbf{D}^{T}\left(\mathbf{D} \mathbf{D}^{T}\right)^{-1}=\mathbf{D}^{T}\left(\mathbf{D}^{+}\right)^{T} \mathbf{D}^{+}=\left(\mathbf{D}^{+} \mathbf{D}\right)^{T} \mathbf{D}^{+}=\mathbf{D}^{+} \mathbf{D} \mathbf{D}^{+}=\mathbf{D}^{+}
$$

Thus, Eq. (35) can be rewritten in the following form

$$
\delta \mathbf{q}=-\mathbf{D}^{T}\left(\mathbf{D D}^{T}\right)^{-1} \boldsymbol{\Phi}\left(\mathbf{q}^{u}\right)
$$


Finally, introducing Eq. (40) into Eq. (31) yields

$$
\mathbf{q}^{c}=\mathbf{q}^{u}-\mathbf{D}^{T}\left(\mathbf{D} \mathbf{D}^{T}\right)^{-1} \boldsymbol{\Phi}\left(\mathbf{q}^{u}\right)
$$

that represents the corrected generalized coordinates in each integration time step. It must be noticed that the kinematic constraint equations at the position level are, in general, nonlinear, then Eq. (41) must be solved iteratively by employing a numerical algorithm, such as the Newton-Raphson method.

In a similar manner, the vector of generalized velocities can be corrected as

$$
\mathbf{v}^{c}=\mathbf{v}^{u}+\delta \mathbf{v}
$$

where $\delta \mathbf{v}$ is the term that has to be added to vector $\mathbf{v}^{u}$ to guarantee that the velocity constraint equations (2) are satisfied, that is

$$
\dot{\boldsymbol{\Phi}}\left(\mathbf{q}^{c}, \mathbf{v}^{c}\right)=\dot{\boldsymbol{\Phi}}\left(\mathbf{q}^{c}, \mathbf{v}^{u}\right)+\delta \dot{\boldsymbol{\Phi}}=\mathbf{0}
$$

The term $\delta \dot{\Phi}$ represents the variation of the velocity constraint equations expressed as

$$
\delta \dot{\Phi}=\frac{\partial \dot{\Phi}}{\partial \mathbf{q}} \delta \mathbf{q}+\frac{\partial \dot{\Phi}}{\partial \dot{\mathbf{q}}} \delta \dot{\mathbf{q}}
$$

The first term of the right-hand side of Eq. (44) is null because at this stage it is assumed that the vector of generalized coordinates is already correct, and, consequently, $\delta \mathbf{q}=\mathbf{0}$. In turn, it is known that the derivative of the velocity constraint equations with respect to vector of generalized velocities is represented by the Jacobian matrix, D. Hence, Eq. (44) is simplified as

$$
\delta \dot{\Phi}=\mathbf{D} \delta \mathbf{v}
$$

Now combining Eqs. (43) and (45) results in

$$
\dot{\Phi}\left(\mathbf{q}^{c}, \mathbf{v}^{u}\right)+\mathbf{D} \delta \mathbf{v}=\mathbf{0}
$$

From Eq. (46) it is possible to obtain the term that corrects the vector of generalized velocities as

$$
\delta \mathbf{v}=-\mathbf{D}^{-1} \dot{\boldsymbol{\Phi}}\left(\mathbf{q}^{c}, \mathbf{v}^{u}\right)
$$

Introducing now Eq. (36) into Eq. (47) results in

$$
\delta \mathbf{v}=-\mathbf{D}^{T}\left(\mathbf{D} \mathbf{D}^{T}\right)^{-1} \dot{\boldsymbol{\Phi}}\left(\mathbf{q}^{c}, \mathbf{v}^{u}\right)
$$

Finally, the substitution of Eq. (48) into Eq. (42) yields

$$
\left.\mathbf{v}^{c}=\mathbf{v}^{u}-\mathbf{D}^{T}(\mathbf{D D})^{T}\right)^{-1} \dot{\Phi}\left(\mathbf{q}^{c}, \mathbf{v}^{u}\right)
$$

that represents the corrected generalized velocities in each integration time step. 


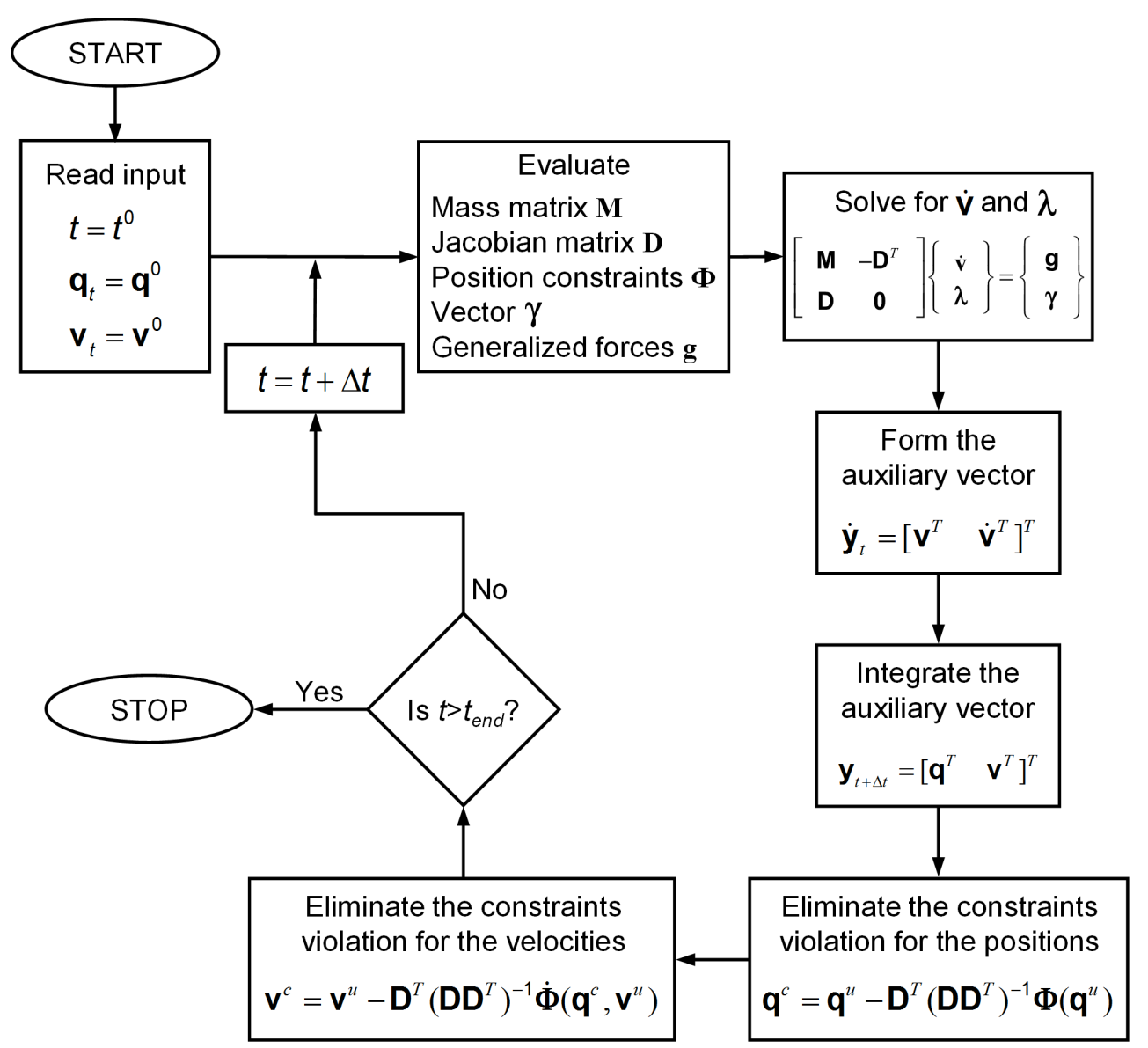

Fig. 3 Flowchart of computational procedure for the solution of the dynamic analysis of constrained multibody systems with the elimination of the constraints violation.

The described methodology can be easily incorporated in the standard method to solve the dynamic equations of motion. The computational strategy for this purpose is illustrated in the flowchart of Fig. 3 and can be summarized by the following steps:

1. Start the analysis by defining the initial conditions of the problem at hand, namely the initial time $t^{0}$, initial positions $\mathbf{q}^{0}$ and initial velocities $\mathbf{v}^{0}$. The initial positions and velocities must satisfy the kinematic constraint equations (1) and (2).

2. Assemble the global mass matrix $\mathbf{M}$, evaluate the Jacobian matrix $\mathbf{D}$, construct the constraint equations $\Phi$, determine the right-hand side of the accelerations $\gamma$, and calculate the generalized force vector $\mathbf{g}$.

3. Solve the linear set of the equations of motion (8) for a constrained multibody system in order to obtain the accelerations $\dot{\mathbf{v}}$ at instant of time $t$ and the Lagrange multipliers $\lambda$.

4. Assemble the vector $\dot{\mathbf{y}}_{t}$ containing the generalized velocities $\mathbf{v}$ and accelerations $\dot{\mathbf{v}}$ for instant of time $t$. 
5. Integrate numerically the velocity and acceleration vectors to obtain the positions and velocities at the instant of time $t+\Delta t$.

6. Evaluate the position constraint violations $\Phi\left(\mathbf{q}^{u}\right)$, compute the corrective term $\delta \mathbf{q}$, by using Eq. (40), and correct the positions vector employing Eq. (41). This step must be repeated if necessary.

7. Evaluate the velocity constraint violations $\dot{\Phi}\left(\mathbf{q}^{c}, \mathbf{v}^{u}\right)$, compute the corrective term $\delta \mathbf{v}$, by using Eq. (48), and correct the velocities vector employing Eq. (49). It must be stated that in this process the matrix $\mathbf{D}$ is evaluated with new values for $\mathbf{q}^{c}$.

8. Update the time variable, go to step 2) and proceed with the process for a new time step, until the final time of analysis is reached.

In opposition to the constraint stabilization approaches, the methodology described above is able to eliminate the violation of the constraints at both position and velocity levels without changing the dynamic equations of motion. In fact, the proposed approach, by means of Eqs. (41) and (49), refines the position and velocities computed solving the DAE system (8), being the violation of constraints satisfied to machine accuracy.

The approach described above does not consider weighting factors to the coordinates and velocities variables. In order to take into account different weighting factors, some works have been proposed to include inertia of bodies, which allow for the adjustments to be made in an inverse manner to the system inertia. The basic idea of this approach is that the more massive bodies are moved the least if the constraints allow that [27, 98, 99, 102]. Thus, Eqs. (41) e (49) are, respectively, substituted by

$$
\begin{gathered}
\mathbf{q}^{c}=\mathbf{q}^{u}-\mathbf{M D}^{T}\left(\mathbf{D} \mathbf{D}^{T}\right)^{-1} \Phi\left(\mathbf{q}^{u}\right) \\
\mathbf{v}^{c}=\mathbf{v}^{u}-\mathbf{M D}^{T}\left(\mathbf{D} \mathbf{D}^{T}\right)^{-1} \dot{\Phi}\left(\mathbf{q}^{c}, \mathbf{v}^{u}\right)
\end{gathered}
$$

At this stage, it must be noticed that, in some circumstances, the evaluation of the generalized inverse matrix given by Eq. (36) can exhibit numerical instabilities and can also be cost from computational point of view. Thus, alternative methodologies can be adopted to overcome these difficulties, namely using the approaches based on the singular value decomposition (SVD) and the Gram-Schmidt Orthogonalization. The interested reader on alternative methodologies is referred to the works by Neto and Ambrósio [32], Udwadia and Phohomsiri [39], Mariti et al. [42], Arabyan and Wu [82], Jalón and Gutiérrez-López [90], Mani and Haug [136], Singh and Likins [137], Kim and Vanderploeg [138], Meijaard [139]. 


\section{Demonstrative examples of application}

A comparative study of several methods to handle the constraints violation is presented in this section. For this purpose, different planar and spatial constrained mechanical systems are considered in order to examine their effectiveness. The different methods are compared to each other in terms of accuracy, conservation of energy and computational efficiency. The mechanical multibody models analyzed, which include open and a closed-loop systems, are a planar four bar mechanism, a spatial five pendulum system, a spatial slider-crank mechanism and a car suspension. It must be stressed that for all the multibody models the initial conditions ensure the position and velocity constraints [102]. All the methods have been implemented in a specialpurpose code, in which the fourth-order Runge-Kutta integrator scheme is utilized in the numerical resolution of the dynamic equations of motion. All the multibody systems are simulated and analyzed for a long time simulation period.

The first example of application is the academic planar four bar mechanism, which is represented in Fig. 4. This multibody system consists of four rigid bodies, including the ground, and four ideal revolute joints. The dimensional and inertia properties of each body together with the set of initial conditions necessary to carry out a dynamic analysis are listed in Table 1 [2]. Table 2 presents the parameters used for the different models, necessary to characterize the problem, and to solve the system dynamics.

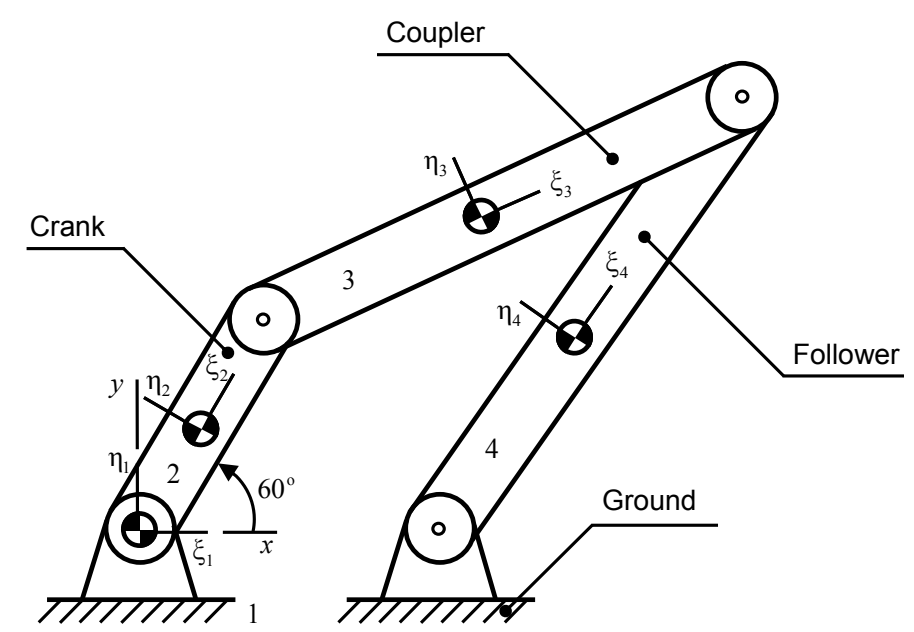

Fig. 4 Multibody model of the planar four bar mechanism.

Table 1 Governing properties for the planar four bar mechanism.

\begin{tabular}{cccccccccc}
\hline Body & $\begin{array}{c}\text { Length } \\
{[\mathrm{m}]}\end{array}$ & $\begin{array}{c}\text { Mass } \\
{[\mathrm{kg}]}\end{array}$ & $\begin{array}{c}\text { Moment of } \\
\text { inertia }\left[\mathrm{kgm}^{2}\right]\end{array}$ & $x[\mathrm{~m}]$ & $y[\mathrm{~m}]$ & $\phi[\mathrm{rad}]$ & $\dot{x}[\mathrm{~m} / \mathrm{s}]$ & $\dot{y}[\mathrm{~m} / \mathrm{s}]$ & $\dot{\phi}[\mathrm{rad} / \mathrm{s}]$ \\
\hline Ground & 2.5 & 0.00 & 0.00 & 0.0000 & 0.0000 & 0.0000 & 0.0000 & 0.0000 & 0.0000 \\
Crank & 2.0 & 1.00 & 0.30 & 0.5000 & 0.8660 & 1.0472 & 0.0000 & 0.0000 & 0.0000 \\
Coupler & 4.0 & 2.25 & 2.00 & 2.8235 & 2.5535 & 0.4332 & 0.0000 & 0.0000 & 0.0000 \\
Follower & 4.0 & 2.20 & 1.35 & 3.5735 & 1.6875 & 1.0042 & 0.0000 & 0.0000 & 0.0000 \\
\hline
\end{tabular}


Table 2 Parameters utilized for the dynamic simulations of the planar four bar mechanism.

\begin{tabular}{llll}
\hline Integration time step & $1 \times 10^{-3} \mathrm{~s}$ & Penalty $-\alpha$ & $1 \times 10^{7}$ \\
Integrator algorithm & Runge-Kutta $-4^{\text {th }}$ order & Penalty $-\omega$ & 10 \\
Baumgarte $-\alpha, \beta$ & 5 & Penalty $-\mu$ & 1 \\
\hline
\end{tabular}

Figure 5 shows the plots of the constraints violation $\left(\boldsymbol{\Phi}^{T} \boldsymbol{\Phi}\right)$ resulting from the dynamic simulation of the planar four bar mechanism. In this particular example, six methods are utilized to solve the system dynamics, namely the standard Lagrange multipliers method, the Baumgarte stabilization approach, the augmented Lagrangian formulation, the index-1 augmented Lagrangian method, the coordinate partitioning method and the direct correction methodology presented in the previous section. It should be highlighted that different scales are used for results plotted in Figs. 5a and 5b, in order to clearly demonstrate the influence of the method utilized. From the results shown in Fig. 5a, as expected, it can be observed that when the standard Lagrange multipliers method is considered, the violation of constraints grows indefinitely with the time $[2,23,31]$. However, when the Baumgarte stabilization method is considered, the response is slightly different. In fact, with the Baumgarte approach, the constraints violation does not growth with time, instead it tends to stabilize or stay under control, as it can be observed from Fig. 5b. Furthermore, the augmented Lagrangian formulation exhibits better behavior when compared to the previous analyzed approaches. Finally, it can be observed that the index-1 augmented Lagrangian formulation, the coordinate partitioning method and the direct correction approach completely eliminate the violation of constraints, visible the diagrams of Fig. 5b. Indeed, with the methodology described in the previous section, the average of the constraints violation is of order $1.0 \times 10^{-18}$. It should be noticed that for the index-1 augmented Lagrangian formulation, the coordinate partitioning method and the direct correction approach the system performance in terms of constraints violation is coincident, and, consequently, the corresponding lines are overlapped in bottom of the plots in Fig. 5. Finally, it must be stressed that a similar analysis and the same conclusions can be drawn from the diagrams plotted in Figs. $6 \mathrm{a}$ and $6 \mathrm{~b}$, corresponding to velocity constraints violation. Again, the different scales are utilized for the results plotted in Figs. $6 a$ and 6 b, with the purpose to clearly observe the influence of the methods used to resolve the system dynamics on the constraints violation. In general, the results plotted for the dynamic simulation of the planar four bar mechanism are in line with those published in $[2,7,31,32,42,70,78,127]$.

Finally, it must be stated that the proposed approach to deal with the constraints violation changes all the coordinates to fulfill the constraint equations after the integration process, by using Eqs. (40) and (41). This way of handle the constraints violation at the position level 
involves the numerical resolution of nonlinear systems of equations, according to the minimum norm condition. In contrast, the Coordinate Partitioning Method evaluates the dependent positions and velocities with the knowledge of the independent stets and the constraint equations. This way of solving the problem can arise some numerical difficulties and efficiency, as it has been addressed in the thematic literature [32, 80,91]. Nevertheless, the proposed approach is quite efficiency from computational point of view, because for the simulations performed within the goals of the present work, in general, no more the 2 iterations are needed to obtained a good convergence of the method. However, special attention to consistency associated with numerical integration process must be given.

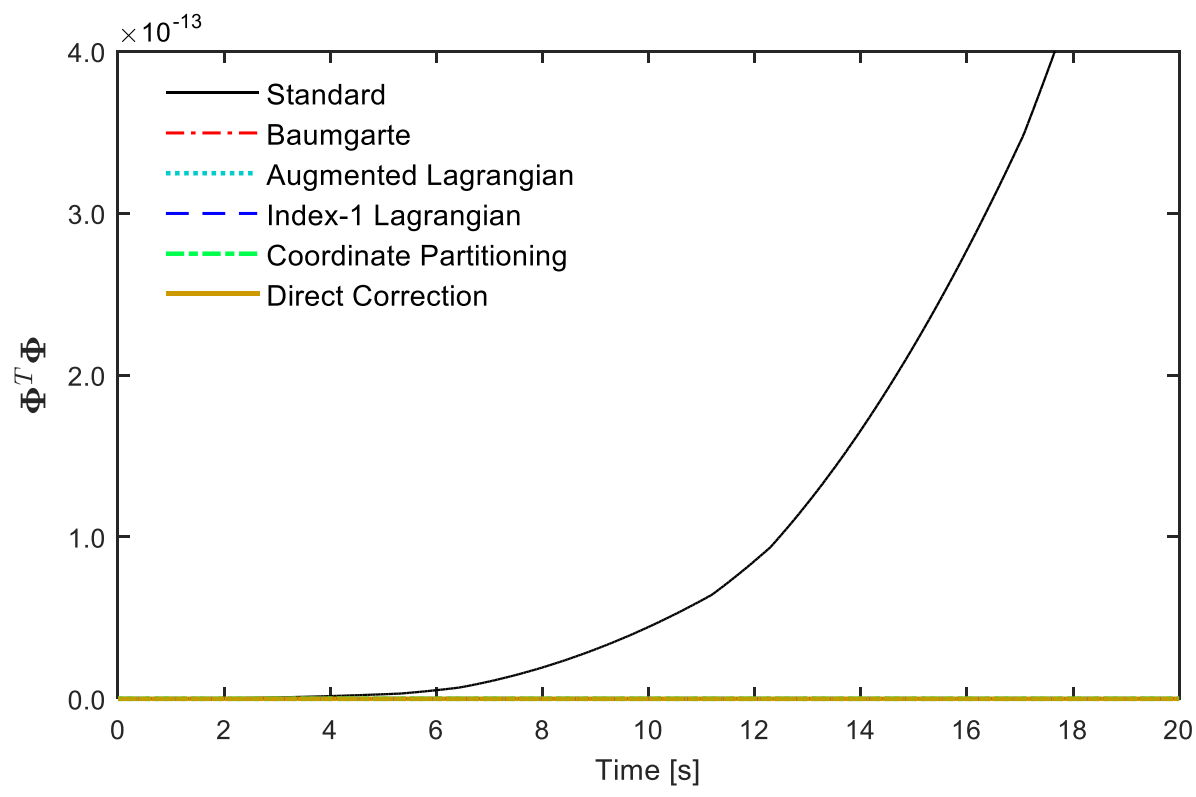

(a)

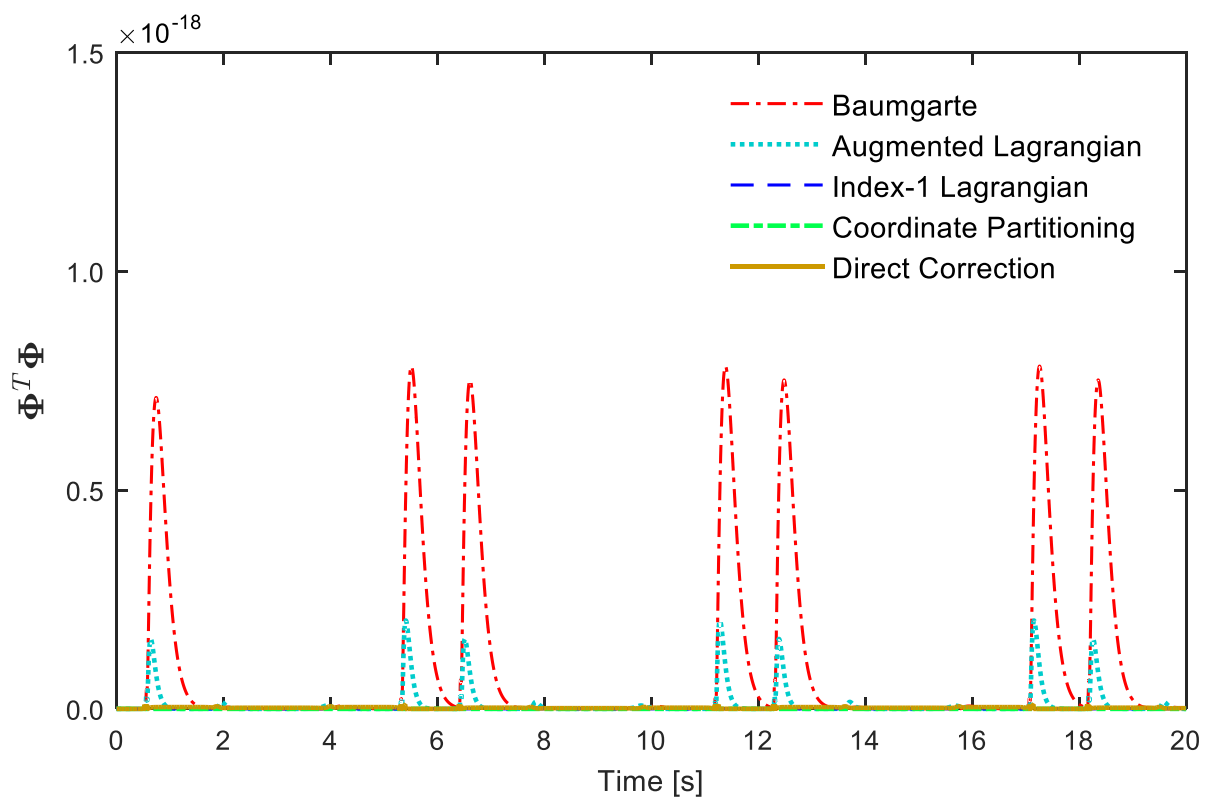

(b)

Fig. 5 Position constraints violation of the planar four bar mechanism. 


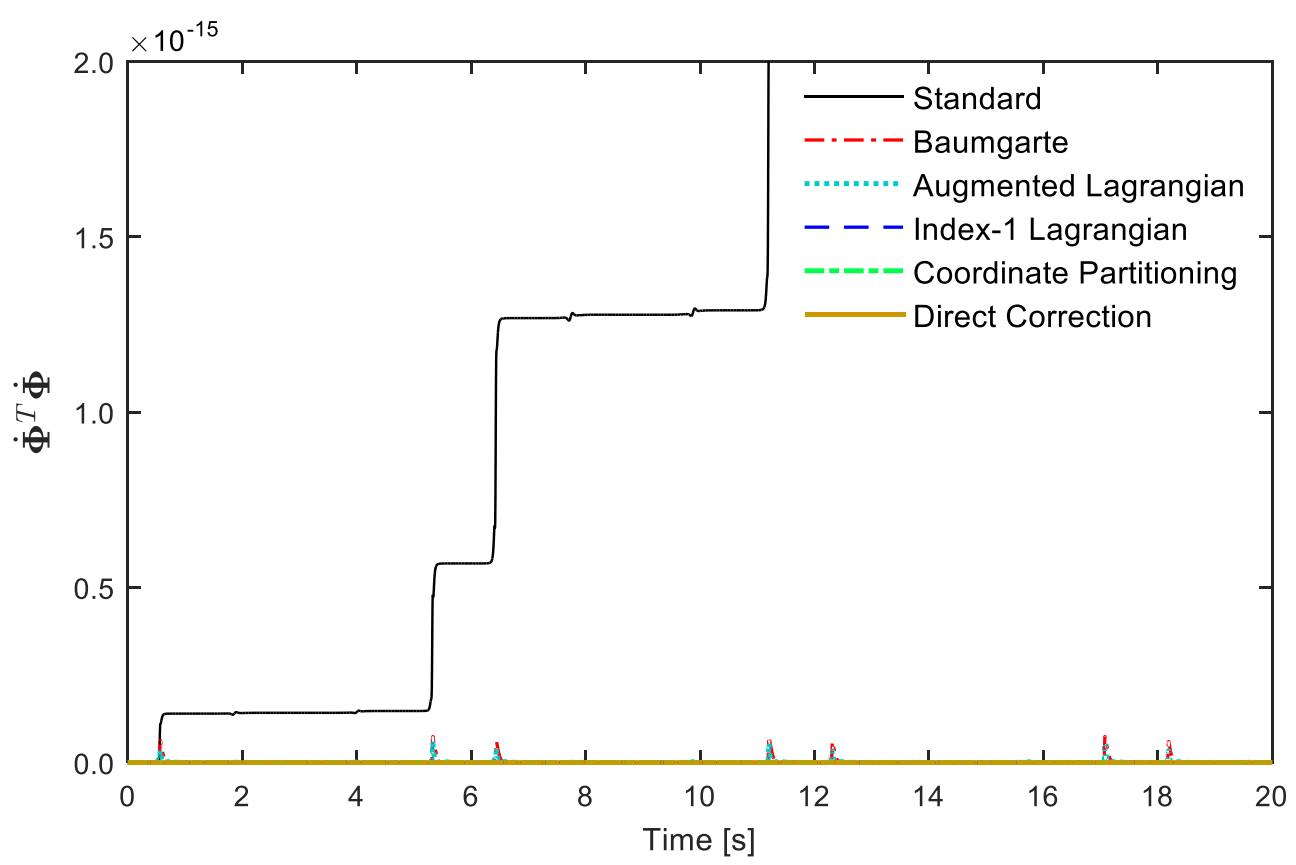

(a)

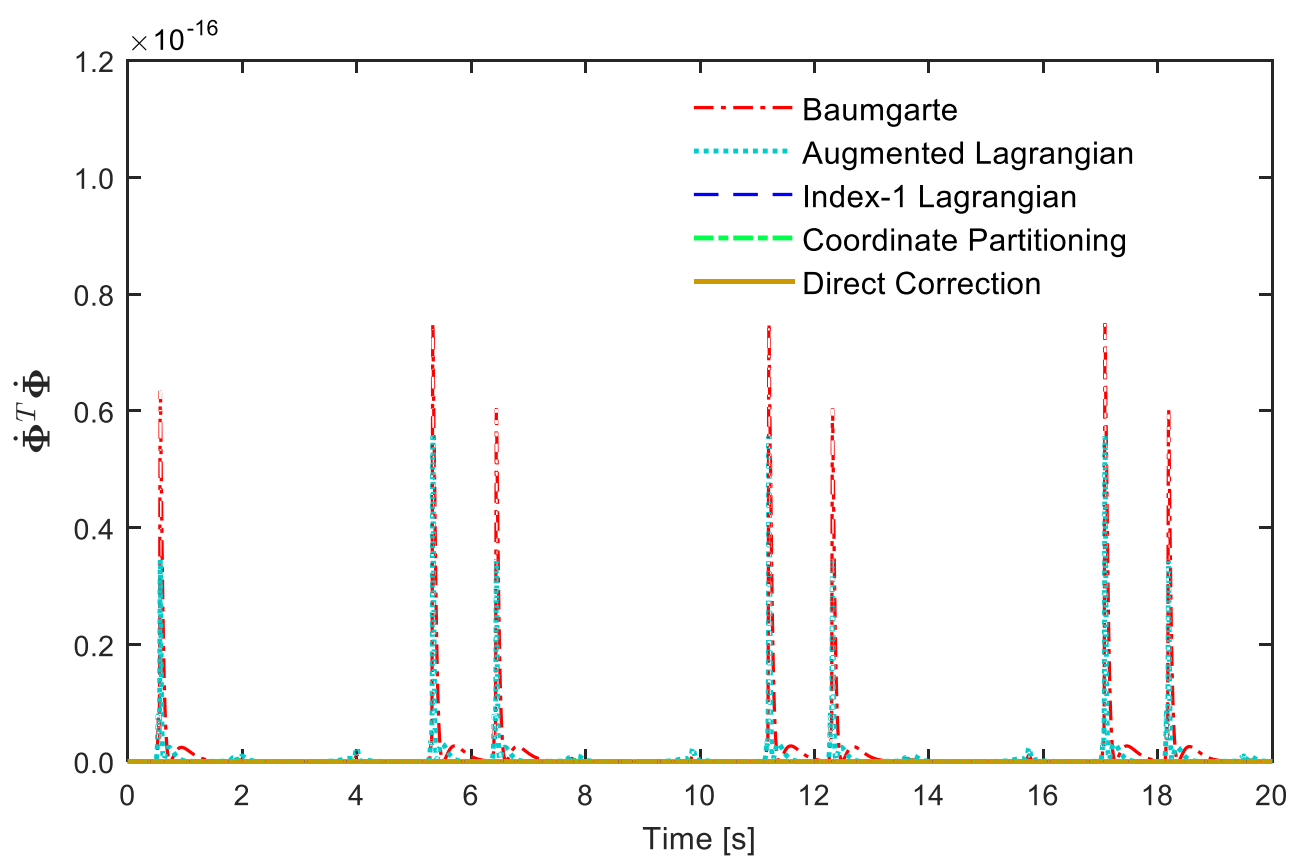

(b)

Fig. 6 Velocity constraints violation of the planar four bar mechanism.

Figure 7 displays the variation of the mechanical energy for the planar four bar mechanism, which is a conservative system, that is [18]

$$
\Delta E=E-E^{0}
$$

where $E$ denotes the sum of the potential and kinetic energies of the mechanism, and $E^{0}$ represents the initial mechanical energy. From the analysis of the mechanical energy variation, it 
can be observed that the coordinate partitioning method and the direct correction approach are more consistent in terms of total mechanical energy conservation. For the coordinate partitioning method and the direct correction approach the system performance is coincident, and, consequently, the corresponding lines are overlapped in bottom of the plots in Fig. 7.

In contrast, for the remaining methods the mechanical energy drifts during the numerical simulations. This numerical problem can be associated with the integrator scheme considered, that is, the Runge-Kutta algorithm. In fact, this particular issue of energy overestimation can be catastrophic for long time simulations of multibody systems.

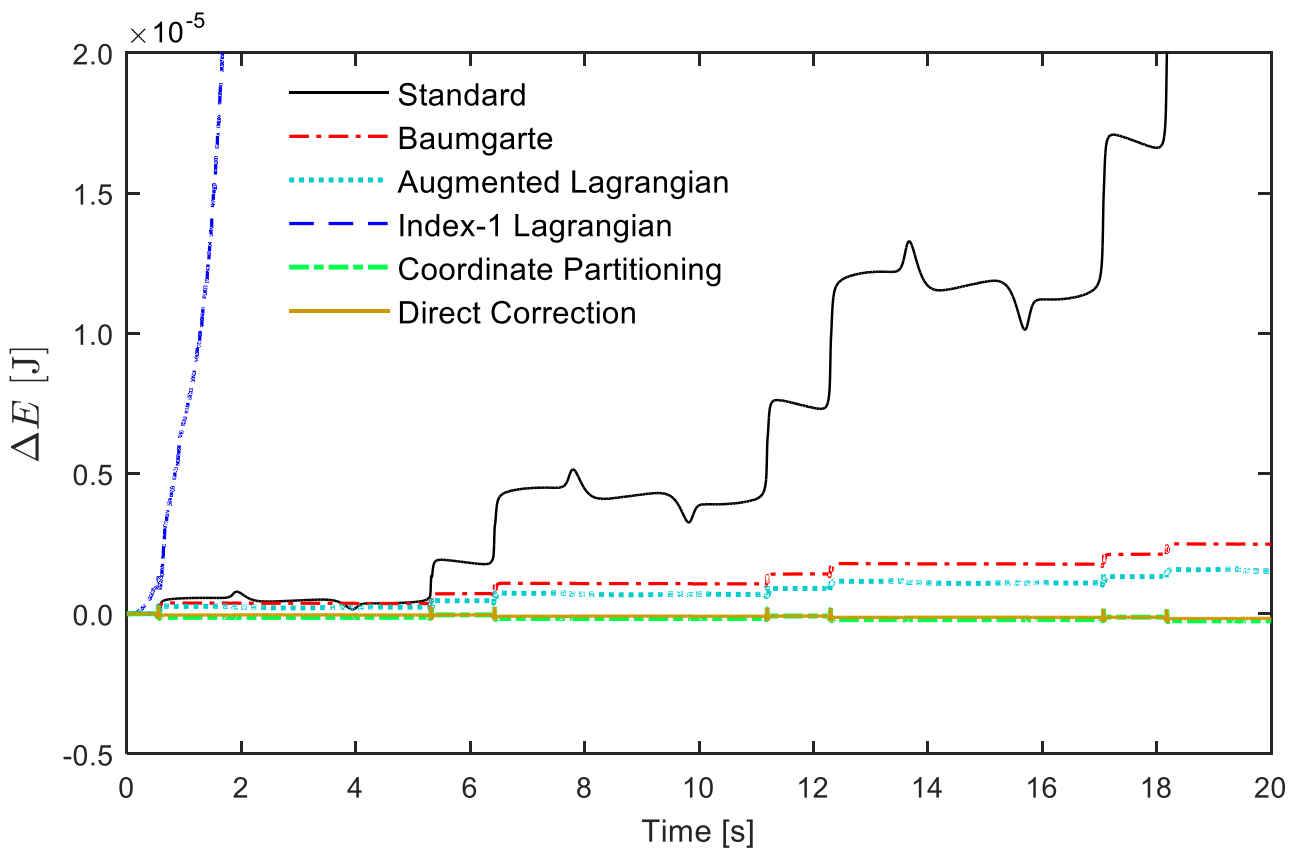

Fig. 7 Variation of the mechanical energy for the planar four bar mechanism.

The efficiency of the different methods utilized to solve the dynamic equations of motion for the planar four bar mechanism are presented in Fig. 8. As it was expected, the standard Lagrange multipliers method is more efficient approach. Moreover, the augmented Lagrangian formulation as well as the index-1 augmented Lagrangian approach present a similar behavior. These last two methods exhibit better performance when compared with the Baumgarte stabilization approach. The best approaches in terms of computational efficiency for this particular example of application are the coordinate partitioning method and the direct correction approach, as it can be seen in the chart of Fig. 8. Indeed, the direct correction methodology described in this present work is more efficient among all the methods to handle the problem 
associated with the constraints violation. Finally, it must be noted that, the response of the system can be affected by the values selected for the penalty parameters [7, 23, 31].

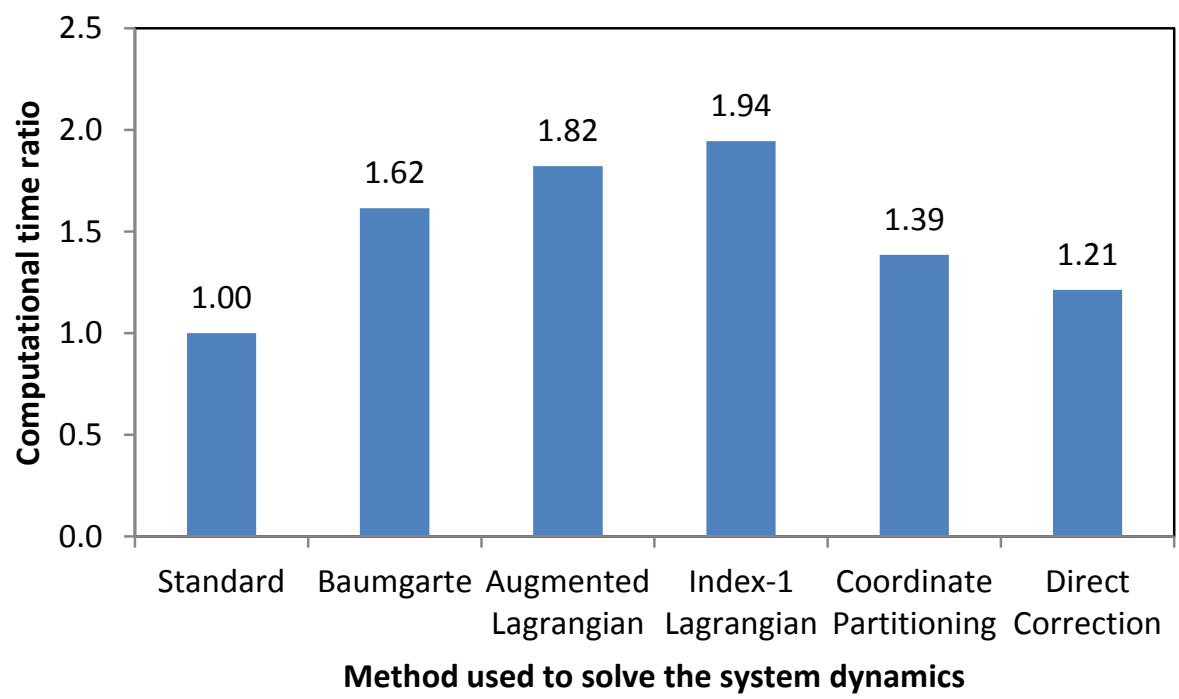

Fig. 8 Computation time ratio for the different methods for the planar four bar mechanism.

The second demonstrative example of application is the spatial five bar pendulum [7, 103-105]. This mechanical system is composed by five rigid arms connected by five revolute joints, as it is illustrated in Fig. 9. Each arm is considered as a prismatic homogeneous bar with square section of $0.1 \times 0.1 \mathrm{~m}^{2}$, unit length and unit mass. This five bar multibody system is modeled with 35 coordinates, three Cartesian coordinates and four Euler parameters per body, and 25 kinematic constraints associated with the revolute joints, yielding a system with five degrees of freedom $[2,8]$. Initially, the five bar pendulum is resting in the configuration represented in Fig. 9. The system is then released from this initial arrangement only subjected to gravity, which is taken as acting in the negative $z$-direction. The magnitude of the gravitational acceleration is equal to $9.81 \mathrm{~m} / \mathrm{s}^{2}$. Again, the Runge-Kutta algorithm is utilized to solve the equations of motion for a period of time equal to $10 \mathrm{~s}$ with an integration time step of $1 \times 10^{-3} \mathrm{~s}$.

Figures 10 and 11 show the constraints violation at the position and velocity levels for the five bar pendulum, when the Baumgarte stabilization approach, the augmented Lagrangian formulation, the index-1 augmented Lagrangian approach and the direct correction methodology are used in the resolution of the dynamic equations of motion. As it was expected, with the stabilization methods, the constraints violation is kept under control during the computational simulations. In turn, when the direct correction approach, described in the previous section, is utilized, the constraint violation is eliminated as it can be observed in the plots of Figs. 10 and 
11. In fact, with the described approach the average of the constraints violation is of order $1.0 \times 10^{-18}$. In general, the outcomes obtained here are in line with those available in the literature [7, 23, 95, 103]. Finally, Fig. 12 the computation time consumed in the dynamic simulations for the five bar pendulum, which can be utilized to have an idea about the computational efficiency of the different methods considered to solve the system dynamics.

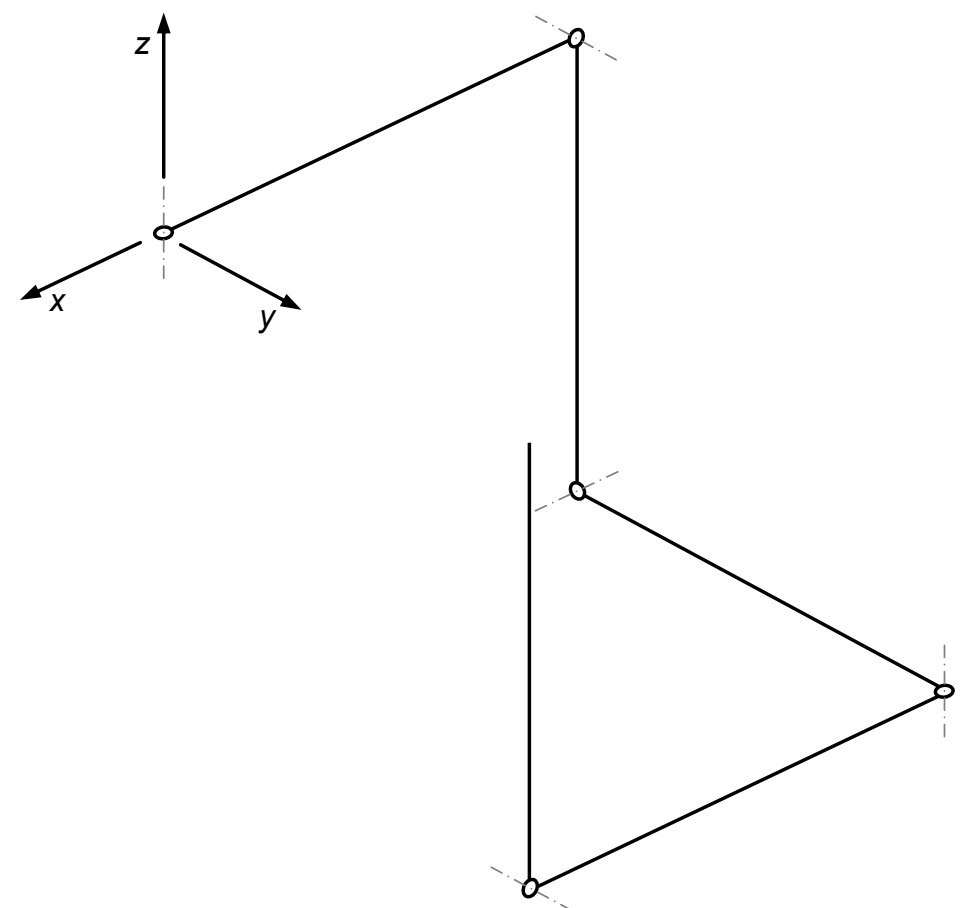

Fig. 9 Multibody model of the spatial five bar pendulum.

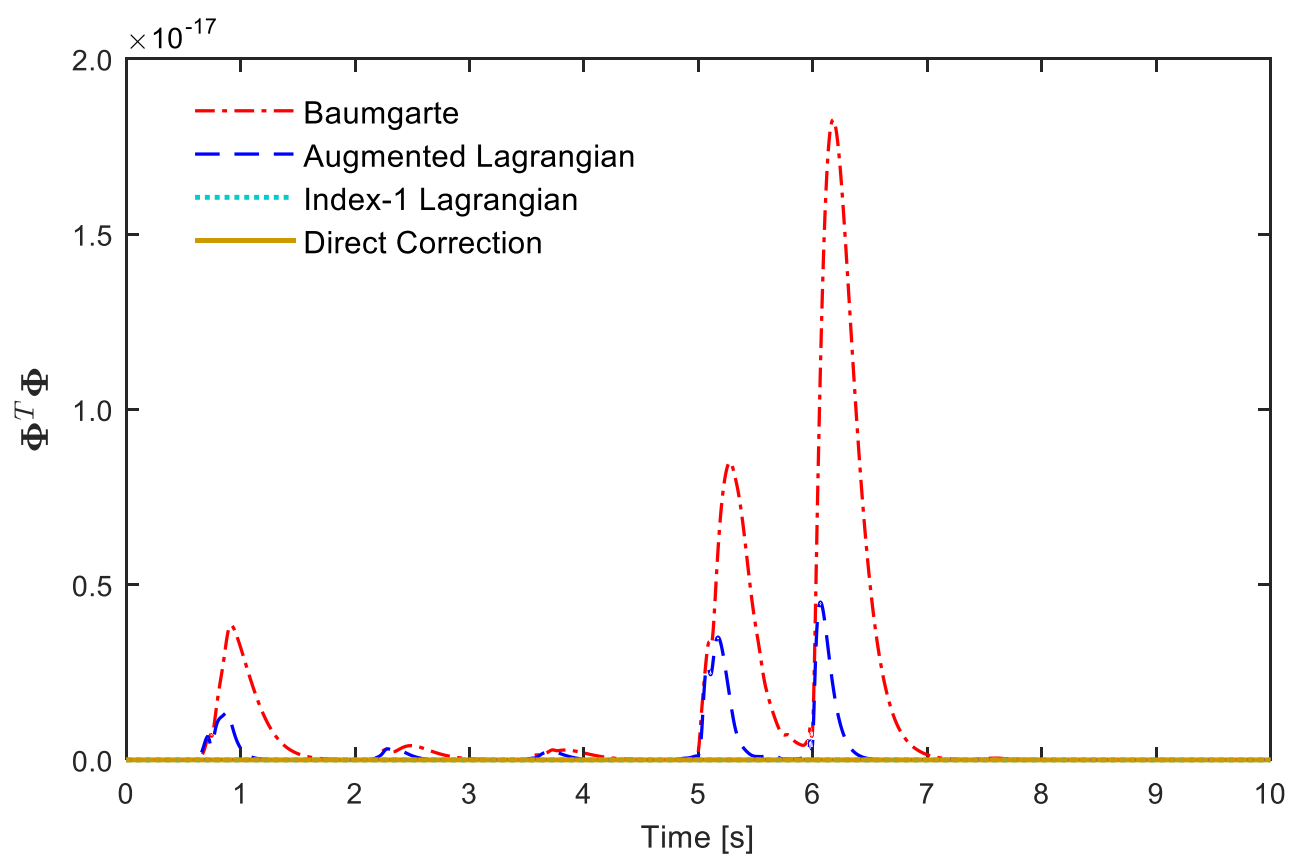

Fig. 10 Position constraints violation of the spatial five bar pendulum. 


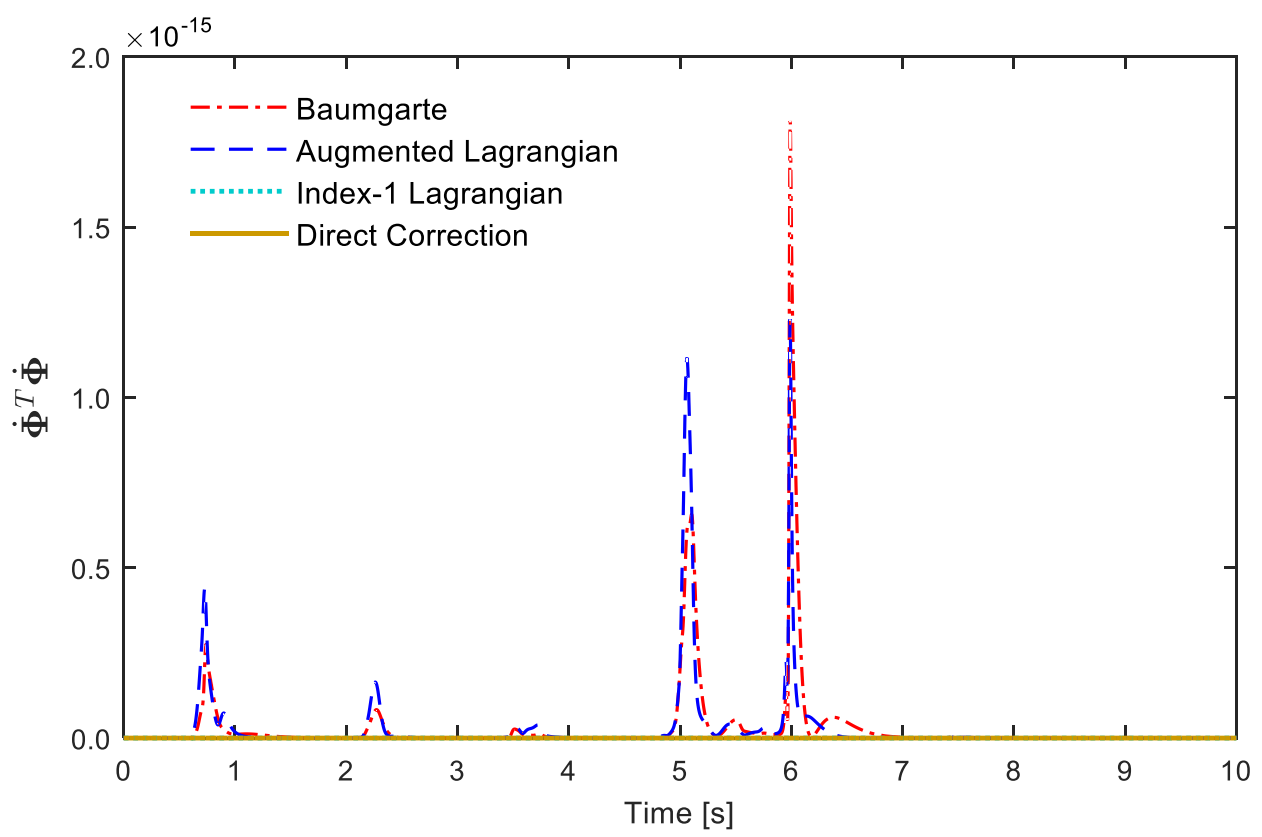

Fig. 11 Velocity constraints violation of the spatial five bar pendulum.

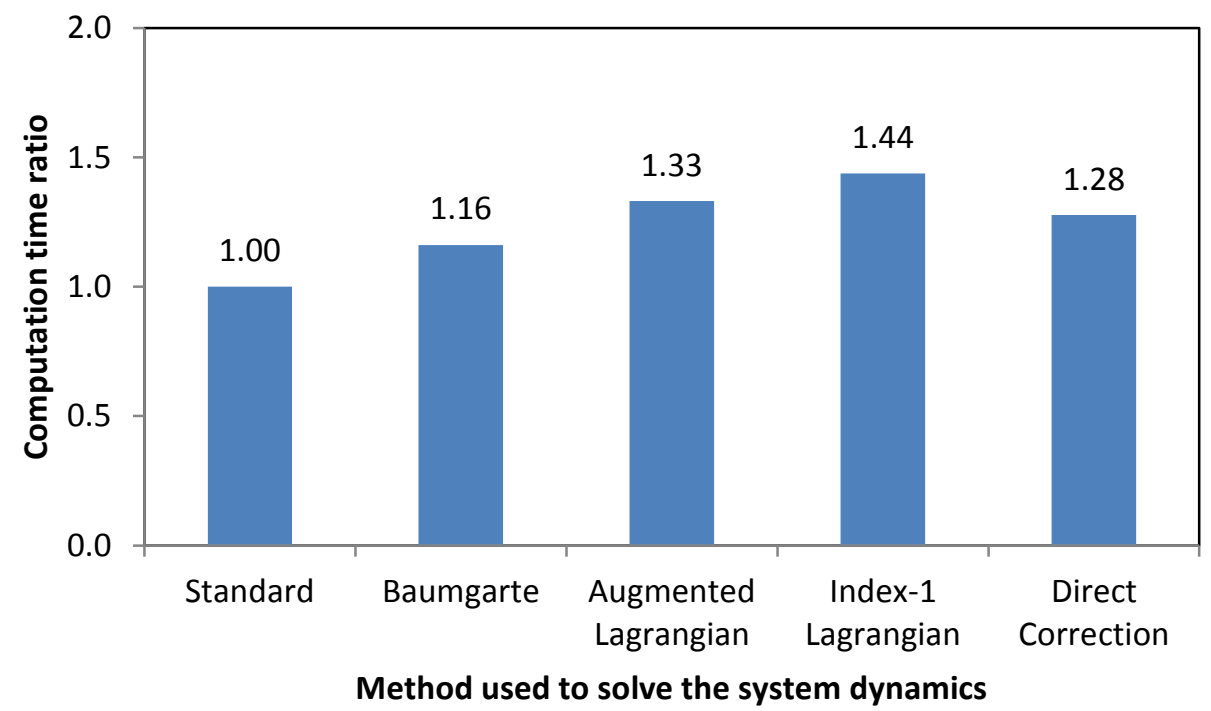

Fig. 12 Computation time ratio for the different methods for the spatial five bar pendulum.

The third example of application utilized to demonstrate the effectiveness of the method presented in this work is the well known benchmark problem of the spatial slider-crank mechanism $[4,44,140]$. This mechanical system is composed by three rigid bodies that are constrained by one revolute joint, one spherical joint and one translational joint, as it is illustrated in Fig. 13. The multibody model of this spatial slider-crank mechanism is described by 21 coordinates and 17 kinematic constraints associated with the kinematic pairs [2]. Gravity is acting in the negative $z$-direction. The dimensions and inertia properties of the system's bodies are listed in Table 3. Moreover, the initial conditions necessary to perform the dynamic analysis are presented in Table 4. For this, the Runge-Kutta algorithm is used to solve the equations of motion for a period of time equal to $5 \mathrm{~s}$ with an integration time step of $1 \times 10^{-3} \mathrm{~s}$. 


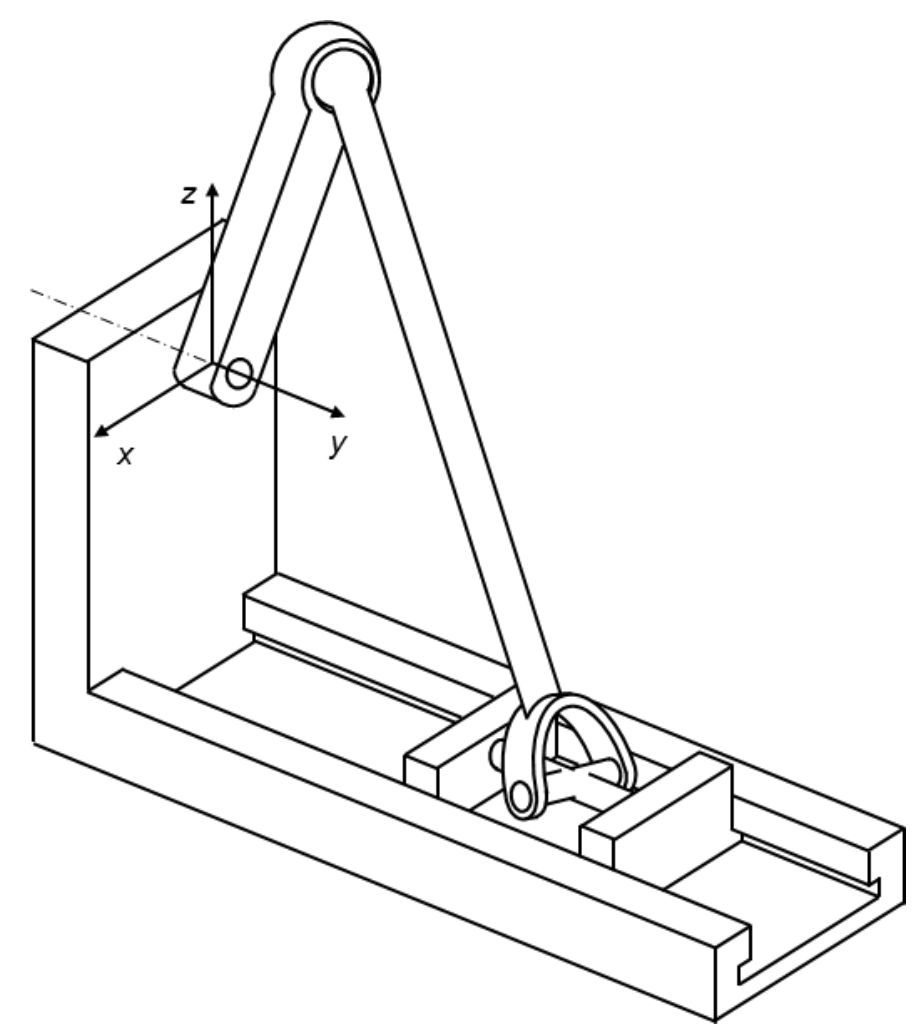

Fig. 13 Multibody model of the spatial slider-crank mechanism.

Table 3 Governing properties for the spatial slider-crank mechanism.

\begin{tabular}{cccccc}
\hline \multirow{2}{*}{ Body } & \multirow{2}{*}{ Length $[\mathrm{m}]$} & Mass $[\mathrm{kg}]$ & \multicolumn{3}{c}{ Moment of inertia $\left[\mathrm{kgm}^{2}\right]$} \\
\cline { 4 - 6 } & 0.10 & 0.12 & $I_{\xi \xi}$ & $I_{n \eta}$ & $I_{\zeta \zeta}$ \\
\hline Crank & 0.29 & 0.50 & 0.00010 & 0.00010 & 0.00001 \\
Connecting rod & - & 0.50 & 0.00010 & 0.00040 & 0.00400 \\
Slider & & &
\end{tabular}

Table 4 Initial conditions for the spatial slider-crank mechanism.

\begin{tabular}{cccccccccccccc}
\hline Body & $x[\mathrm{~m}]$ & $y[\mathrm{~m}]$ & $z[\mathrm{~m}]$ & $e_{0}$ & $e_{1}$ & $e_{2}$ & $e_{3}$ & $v_{x}$ & $v_{y}$ & $v_{z}$ & $\omega_{x}$ & $\omega_{y}$ & $\omega_{z}$ \\
\hline Crank & 0.000 & 0.0000 & 0.0500 & 1.0000 & 0.0000 & 0.000 & 0.000 & -0.3142 & 0.000 & 0.000 & 0.000 & -6.2832 & 0.000 \\
Connecting rod & 0.000 & 0.0735 & -0.0250 & 0.8680 & -0.4966 & 0.000 & 0.000 & -0.3142 & 0.000 & 0.000 & 0.000 & -2.5133 & 0.000 \\
Slider & 0.000 & 0.1470 & -0.1500 & 1.0000 & 0.0000 & 0.000 & 0.000 & 0.0000 & 0.000 & 0.000 & 0.000 & 0.0000 & 0.000 \\
\hline
\end{tabular}

Figures 14 and 15 present the evolution of the constraints violation at the position and velocity levels for the spatial slider-crank mechanism when different methods are utilized to solve the dynamic equations of motion. From the analysis of the diagrams of these figures, it can be drawn that when the standard Lagrange multipliers method is employed, the violation of the constraint equations grows indefinitely with time. In reality, the standard approach based on the Lagrange multipliers technique produces unacceptable results because the kinematic equations are rapidly violated due to the inherent errors and instability that develop during computations. In turn, with the stabilization methods, the system's behavior is significantly different, in the measure that the level of the constraints violation is kept under control during the computational 
simulations. In fact, with the Baumgarte approach, the augmented Lagrangian formulation and the index-1 augmented Lagrangian approach experience tells that the numerical results do not diverge from the exact solution, but oscillate around it. Magnitude and frequency of the oscillation depend on the values of the penalty parameters used [4, 7, 23, 31]. Finally, when the methodology described in this work is utilized in the resolution of the dynamic equations of motion, the constraints violation is eliminated, as it can be observed in Figs. 14 and 15, being the average of the constraints violation of order $1.0 \times 10^{-18}$. The computation time consumed in the dynamic simulation of the spatial slider-crank mechanism is plotted in Fig. 16. In a similar manner to the previous examples, the plots can be considered to have an idea of the computational efficiency associated with the use of different methods.

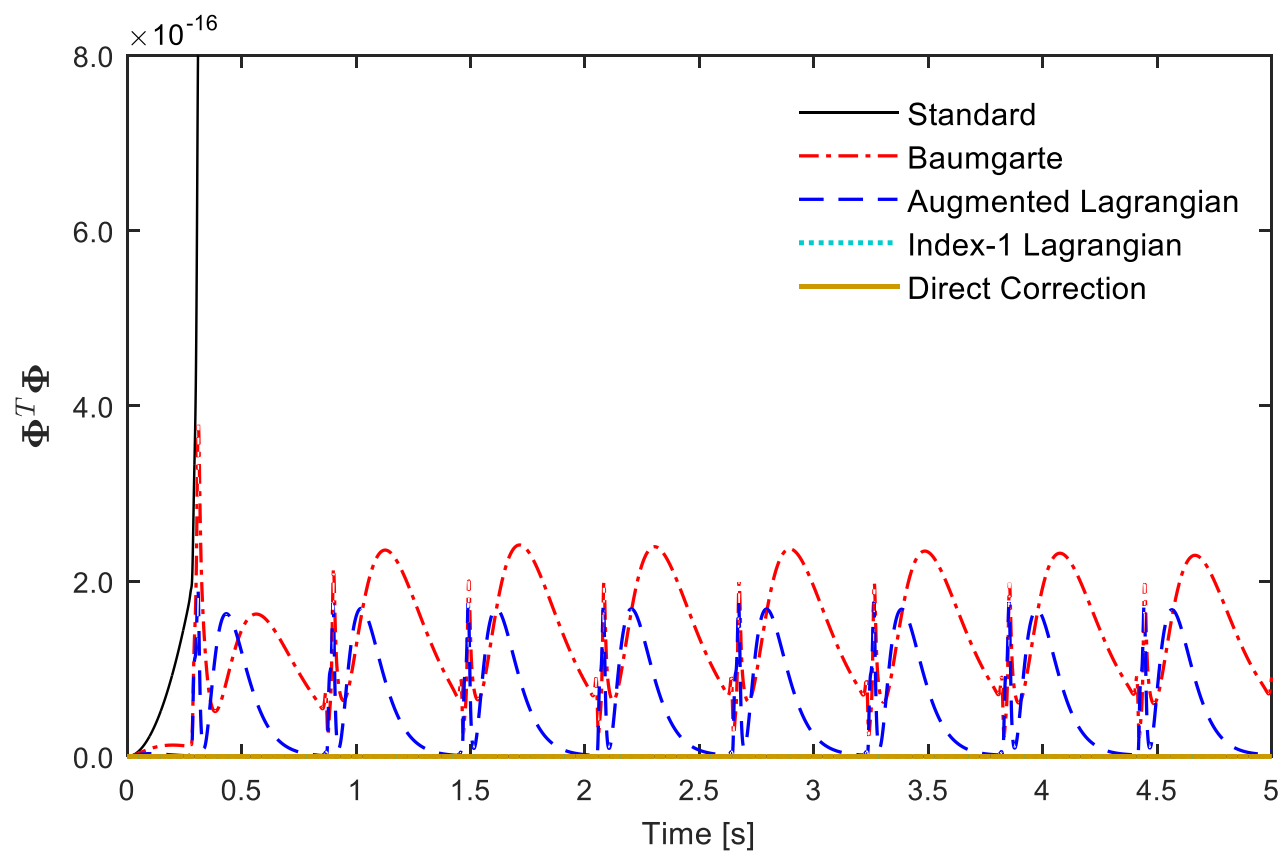

Fig. 14 Position constraints violation of the spatial slider-crank mechanism. 


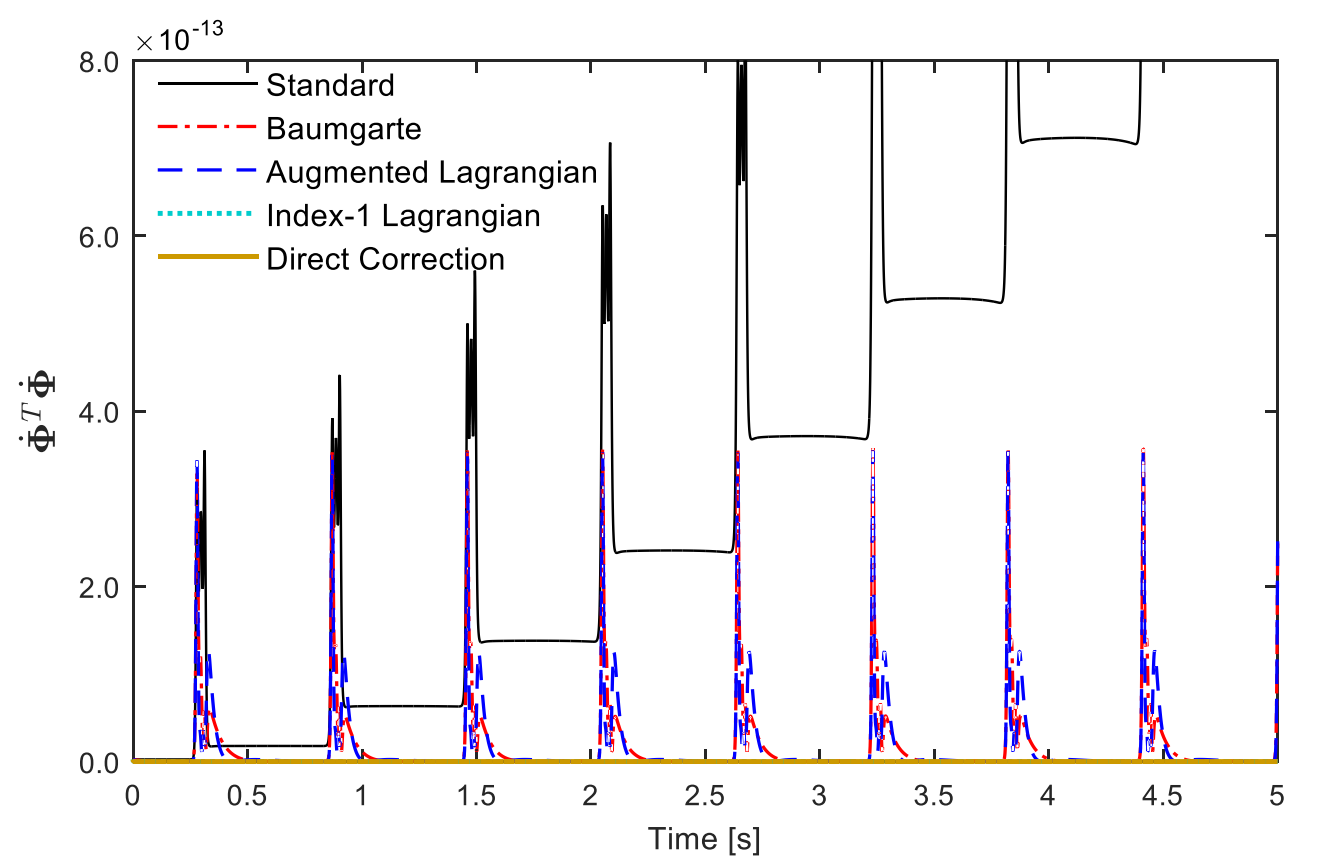

Fig. 15 Velocity constraints violation of the spatial slider-crank mechanism.

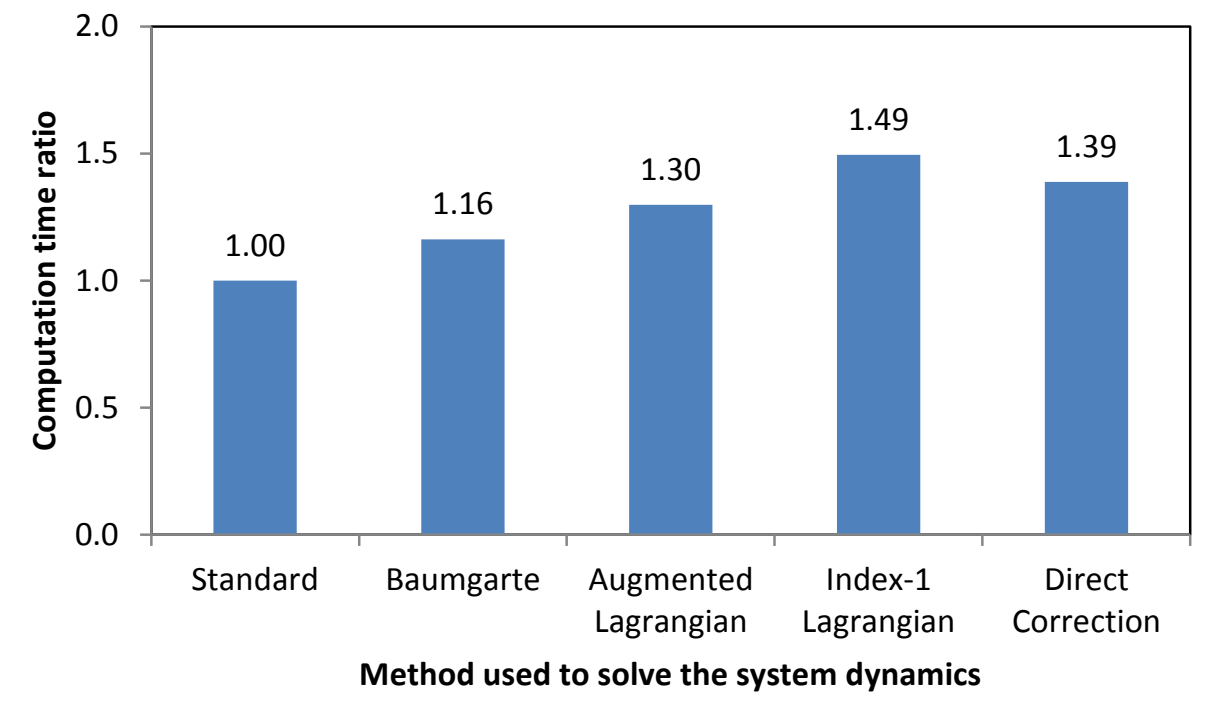

Fig. 16 Computation time ratio for the different methods for the spatial slider-crank mechanism.

The last example of application considered to assess the accuracy and efficiency of the different methodologies presented to solve the dynamic equations of motion is the right front suspension of a car that passes over a bump. The European automobile industry proposed the 4x4 BOMBARDIER ILTIS vehicle as a benchmark problem to validate multibody dynamic codes [141]. As stated, only the right front suspension was modeled for the purpose of this study, as depicted in Fig. 17. The system is constituted by four bodies (the wheel with hub and brake 
assembly, the A-arm, the leaf spring, and the tie rod), which consists in 28 variables, and two revolute joints and three spherical joints resulting in 19 constraints.

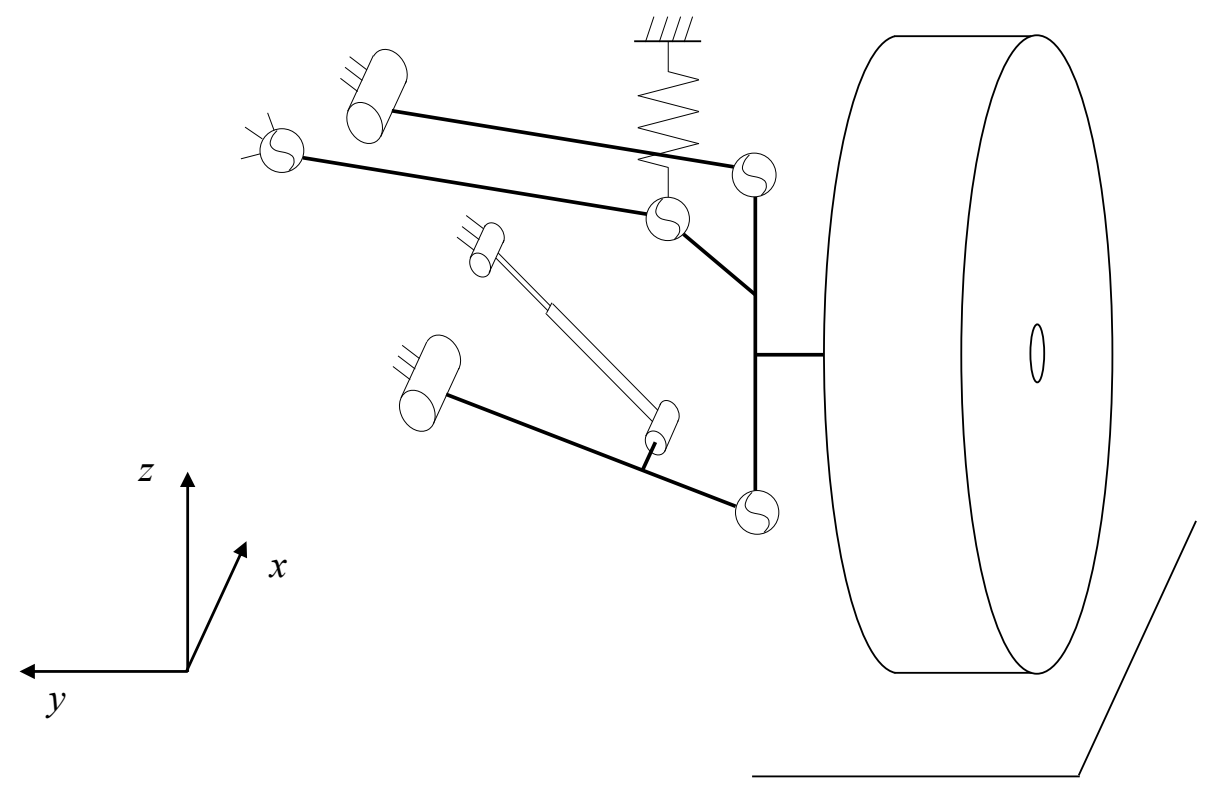

Fig. 17 Multibody model of the right front suspension of a car.

In order to perform a dynamic simulation, the inertial properties of each body are listed in Table 5, the masses and moments of inertia of the bodies not mentioned are to be neglected. Furthermore, the initial positions of this model are presented in Table 6, and the suspension is moving with constant velocity of $5 \mathrm{~m} / \mathrm{s}$ in the $x$ direction.

Table 5 Inertial properties of the car suspension.

\begin{tabular}{ccccc}
\hline \multirow{2}{*}{ Body } & \multirow{2}{*}{ Mass $[\mathrm{kg}]$} & \multicolumn{3}{c}{ Moment of inertia $\left[\mathrm{kg} \mathrm{m}^{2}\right]$} \\
\cline { 3 - 5 } & 57.35 & $I_{\xi \xi}$ & $I_{\eta \eta}$ & $I_{\zeta \zeta}$ \\
\hline Wheel w/ hub and brake & 57.35 & 1.2402 & 1.9080 & 1.2402 \\
A-arm & 6.0 & 0.052099 & 0.023235 & 0.068864 \\
\hline
\end{tabular}

Table 6 Initial conditions for the spatial slider-crank mechanism.

\begin{tabular}{cccccccc}
\hline Body & $x[\mathrm{~m}]$ & $y[\mathrm{~m}]$ & $z[\mathrm{~m}]$ & $e_{0}$ & $e_{1}$ & $e_{2}$ & $e_{3}$ \\
\hline Wheel w/ hub and brake & 0.0000 & -0.6150 & 0.3560 & 1.0000 & 0.0000 & 0.0000 & 0.0000 \\
A-arm & 0.0000 & -0.4155 & 0.2655 & 0.9934 & 0.1143 & 0.0000 & 0.0000 \\
Leaf spring & 0.0000 & -0.3233 & 0.5655 & 0.9947 & 0.1030 & 0.0000 & 0.0000 \\
Tie rod & -0.1400 & -0.2590 & 0.5655 & 0.9959 & 0.0902 & 0.0000 & 0.0000 \\
\hline
\end{tabular}

The forces acting on the suspension model have a wide range of origins. The most basic are the gravitational force due its own weight. The system contains also a shock absorber which is linked to the A-arm providing an elastic and a damping force, respectively, given by 


$$
\begin{gathered}
F_{S}=-4.0092 \times 10^{6}+2.8397 \times 10^{7} x-6.7061 \times 10^{7} x^{2}+5.2796 \times 10^{7} x^{3} \\
F_{D}= \begin{cases}-416.42+1844.3 \dot{x} & \text { if } \dot{x}<-0.2 \\
9945.627 \dot{x}+33955.72 \dot{x}^{2}-59832.25 \dot{x}^{3}-395651.0 \dot{x}^{4} & \text { if }-0.2<\dot{x}<0.21 \\
1919.1638+1634.727 \dot{x} & \text { if } \dot{x}>0.21\end{cases}
\end{gathered}
$$

where $x$ is the distance between the two ends, and $\dot{x}$ denotes the deformation velocity.

Moreover, a leaf spring is also modeled with two different stiffness, therefore, for deformations smaller than $14.5 \mathrm{~cm}$, it behaves as linear spring of $35900 \mathrm{~N} / \mathrm{m}$. For higher deformations, a bump stop contact occurs, and a second spring is activated with a stiffness of $10^{7}$ $\mathrm{N} / \mathrm{m}$. Finally, the tire is modeled as a linear spring with an unloaded radius of $0.364325 \mathrm{~m}$, and a stiffness of $460000 \mathrm{~N} / \mathrm{m}$. The tire passes over a bump with the following profile

$$
z_{\mathrm{B}}= \begin{cases}0 & \text { if } x_{\mathrm{B}}<15 \\ 0.1\left[1-\cos \left(\frac{2 \pi\left(x_{\mathrm{B}}-15\right)}{5}\right)\right] & \text { if } 15<x_{\mathrm{B}}<20 \\ 0 & \text { if } x_{\mathrm{B}}>20\end{cases}
$$

where $x_{\mathrm{B}}$ represents the horizontal position of the wheel center, and $z_{\mathrm{B}}$ denotes vertical position the deformation velocity.

The dynamic simulation is performed for $6 \mathrm{~s}$, and a Runge-Kutta algorithm is implemented with a step size of $10^{-3} \mathrm{~s}$. The initial position of the system does not correspond to the static equilibrium of the suspension, therefore, it oscillates until the equilibrium is reached. The system is excited when passes over the bump and the leaf spring hits the bump contact stop, as can be seen in Fig. 19 by the vertical position of wheel center.

The violation of constraints at position and velocity levels for different methods are displayed in Figs. 18 and 19. From the previous examples of application, it was shown that the standard Lagrange multipliers technique rapidly violates the kinematic constraints, therefore, it was withdrawn from this comparison. The results of the remaining methodologies corroborate what was obtained for the previous cases. The Baumgarte method and augmented Lagrangian approach present a stabilization of the constraints, either in the initial oscillations or when it passes over the bump. In both situations, the stabilization of the augmented Lagrangian formulation is faster. In turn, when the index-1 Lagrangian formulation or the corrective approach are implemented, the violation of the constraints are completely vanished, as depicted in Figs. 18 and 19 where both lines are overlapped. The magnitude of the constraints vectors for these two methodologies can be neglected, since they are of order of $10^{-25}$ and $10^{-32}$ for the 
index-1 Lagrangian formulation and the corrective technique presented in this work, respectively. In what concerns to the computation time, the results are compared to the standard approach, and the index-1 Lagrangian projection method is the most time consuming, while the direct correction approach is slightly faster. The stabilization methods, i.e., the Baumgarte method and the augmented Lagrangian approach, are even faster, as shown in Fig. 20.

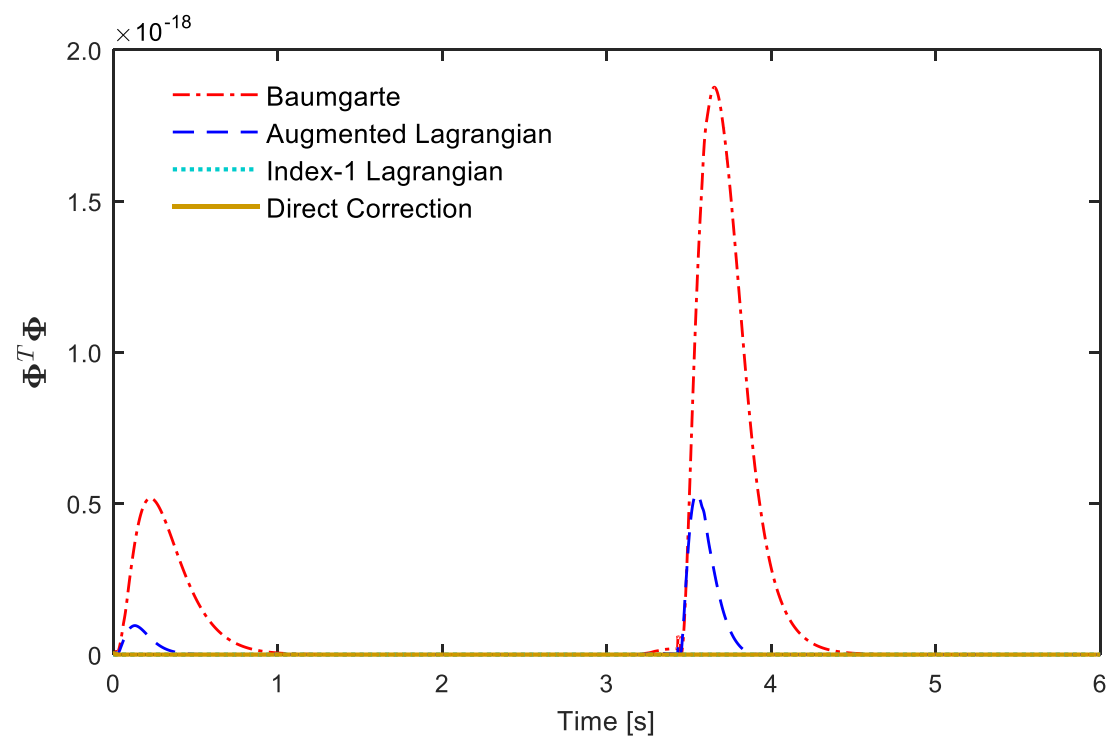

Fig. 18 Position constraints violation of the right front suspension of a car.

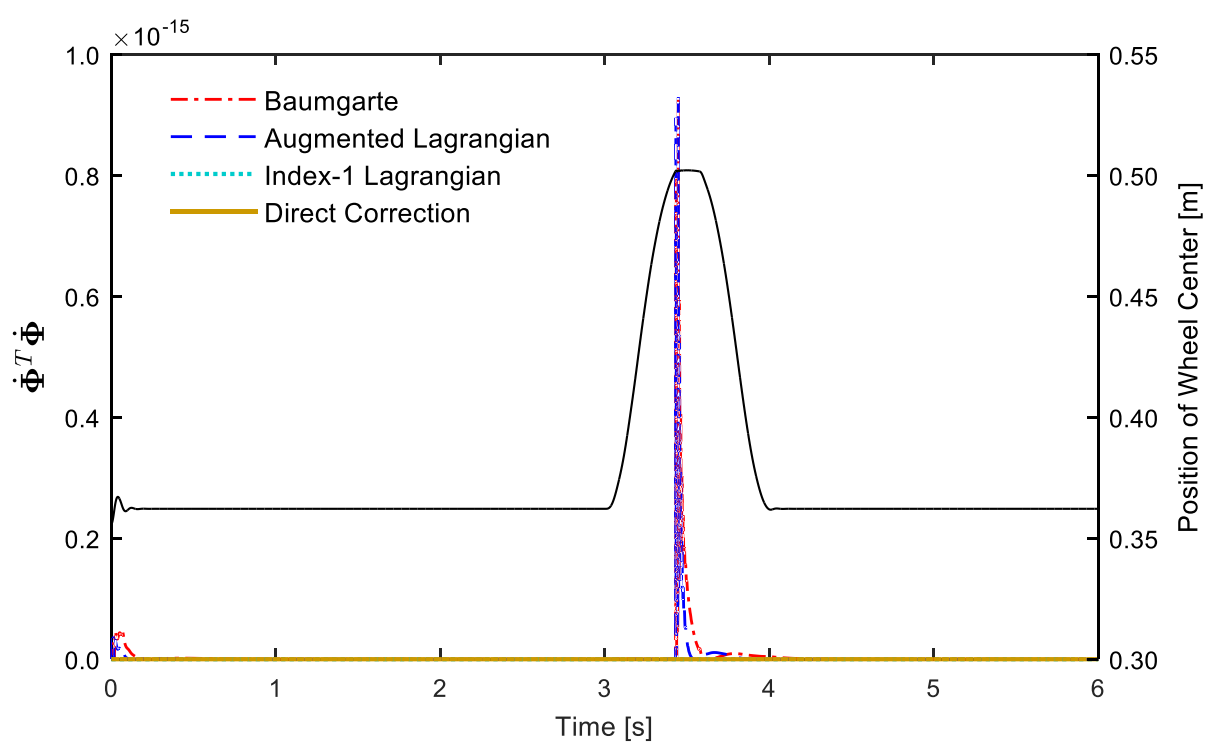

Fig. 19 Velocity constraints violation of the right front suspension of a car. 


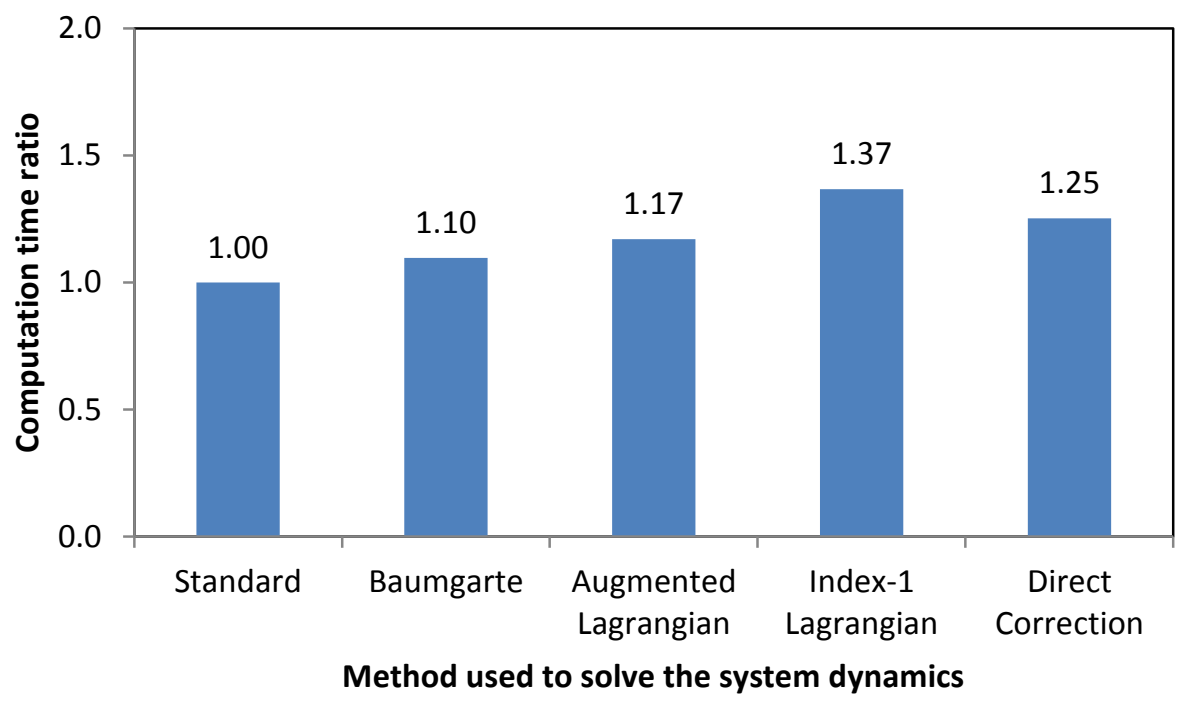

Fig. 20 Computation time ratio for the different methods for the right front suspension of a car.

\section{Conclusions and discussion}

The classic problem of the constraints violation in forward dynamics of mechanical multibody systems has been investigated in the present work. In the sequel of this process, the fundamental characteristics of available methods to handle the problem of constraints violation in multibody dynamics have been analyzed and discussed. Particular emphasis has been given to the Baumgarte stabilization approach, the augmented Lagrangian formulation, the Index-1 Lagrangian formulation and the coordinate partitioning method. Subsequently, an alternative methodology to eliminate the violation of the kinematic constraint equations in the framework of forward dynamics of constrained multibody systems was described. The fundamental idea of the presented approach is to add corrective terms to the position and velocity vectors with the intent to satisfy the corresponding kinematic constraint equations. The corrective terms are evaluated as function of the Moore-Penrose inverse of the Jacobian matrix and of the kinematic constraint equations. The proposed methodology is independent of the integration algorithm utilized and it does not depend on the selection of any ambiguous penalty parameters, since it ensures that the constraint equations are satisfied. In contrast to the constraint stabilization approaches, the presented methodology eliminates the violation of the constraints without changing the dynamic equations of motion.

The validity of the proposed methodology was demonstrated trough the dynamic modeling and analysis of several planar and spatial mechanical multibody systems. The outcomes obtained from the computational simulations performed were compared with the 
results produced with the standard Lagrange multipliers technique, the Baumgarte stabilization approach, the penalty method, the augmented Lagrangian formulation and the coordinate partitioning method. The effectiveness of the methodology described in this work was analyzed and compared in terms of constraints violation, conservation of the total energy and computational efficiency. In a broad sense, it can be stated that the proposed approach is effective in eliminate the constraints violation at both position and velocity levels without penalizing the computational efficiency. With the described methodology, the energy of the system can be over or under estimated because of the modifications associated with the modifications of the positions and velocities constraints. This is a critical and open issue that can be object of further investigation. The efficiency of the described method can be understood by its nature, in the measure that the two-additional blocks are added to the standard solution of the equations of motion. The elimination of the constraints violation for positions requires an iterative procedure, because the corrective terms are dependent on the positions. However, based on the computational tests performed, this process involves at most three iterations to eliminate the constraints violation at the position to an acceptable level. In turn, the constraints violation for velocities are eliminated with a single step, since constraints at the velocity level are linear and the corrective terms are computed as function of the corrected positions performed previously. Finally, it must be stressed that the described methodology is also effective to correct the initial conditions, being the constraint equations satisfied to machine accuracy level.

Finally, it must be highlighted that some difficulties can arise when using the described methodology. First of all, as it was presented previously, the computation of the generalized inverse matrix expressed by Eq. (36) can be instable and highly cost. Alternative approaches to deal with these issues have already been addressed in the literature [32, 39, 42, 90, 136-139]. On the other hand, the process of correction of the positions and velocities by enforcing the corresponding constraint equations does not require the use of a generalized inverse procedure, but its particularization for the case of the minimum norm solution of a compatible undetermined system of linear equations. This particular issue has been analyzed in detail in the seminal work by Jalón and Gutiérrez-López [90]. Additionally, the described methodology can not handle the problem associated with redundant constraints and inconsistency in the units utilized for the coordinates and velocities $[27,78,90,103,113]$. Finally, in opposition to the coordinate partitioning method, the described methodology refines the positions and velocity variables, by means of Eqs. (41) and (49), nevertheless in the correction process they have missed the consistency with the dynamic equations and the numerical integrator. This last issue can have negative consequences in terms of the conservation of the total mechanical energy of the 
systems, as well as in the systems' behavior. Therefore, for future work in the field of studying the problem of constraints violation in multibody systems, the following topics seem to be important: $(i)$ implementation of the presented methodology in more complex mechanical systems, such as automotive systems, heavy machinery; (ii) carrying out an extended investigation on mechanical multibody systems with flexible bodies and joints compliance; (iii) perform a general comparative study in order to obtain a more accurate picture on the large amount of methodologies available to deal with constraint violation; (iv) investigation on multibody systems with redundant constraints and singular configurations.

\section{Acknowledgments}

The first author expresses his gratitude to the Portuguese Foundation for Science and Technology through the PhD grant (PD/BD/114154/2016). This work has been supported by the Portuguese Foundation for Science and Technology with the reference project UID/EEA/04436/2013, by FEDER funds through the COMPETE 2020 Programa Operacional Competitividade e Internacionalização (POCI) with the reference project POCI-01-0145FEDER-006941. The authors also would like to acknowledge the considerable contributions of Professor Javier Cuadrado from University of A Coruña, Spain, for sharing with us some thoughts and material for the numerical examples of application. Finally, the authors are much indebted to the anonymous reviewers for useful comments, recommendations and suggestions.

\section{References}

1. Wittenburg, J.: Dynamics of Systems of Rigid Bodies, B.G. Teubner, Stuttgart, Germany (1977)

2. Nikravesh, P.E.: Computer-Aided Analysis of Mechanical Systems, Prentice Hall, Englewood Cliffs, New Jersey (1988)

3. Shabana, A.A.: Dynamics of Multibody Systems, John Wiley \& Sons, New York (1989)

4. Haug, E.J.: Computer Aided Kinematics and Dynamics of Mechanical Systems V.1: Basic Methods. Allyn \& Bacon, Boston (1989)

5. Schiehlen, W.: Multibody Systems Handbook, Springer-Verlag, Berlin, Germany (1990)

6. Huston, R.L.: Multibody Dynamics. Butterworth-Heinemann, Boston, Massachusetts (1990)

7. Jalón, J.G., Bayo, E.: Kinematic and Dynamic Simulations of Multibody Systems: The Real-time Challenge. Springer Verlag, New York (1994)

8. Flores, P.: Concepts and Formulations for Spatial Multibody Dynamics. Springer, (2015)

9. Amirouche, F.: Computational Methods in Multibody Dynamics. Prentice Hall (1992)

10. Rahnejat, H.: Multi-body dynamics: historical evolution and application. Proceedings of the Institution of Mechanical Engineers, Part C: Journal Mechanical Engineering Science, 214, 149-173 (2000)

11. Udwadia, F.E.: Equations of Motion for Constrained Multibody Systems and their Control. Journal of Optimization Theory and Applications, 127(3), 627-638 (2005)

12. Eberhard, P., Schiehlen, W.: Computational dynamics of multibody systems: History, formalisms, and applications. Journal of Computational and Nonlinear Dynamics, 1, 3-12 (2006)

13. Schiehlen, W.: Research trends in multibody system dynamics. Multibody System Dynamics, 18, 3-13 (2007)

14. Nikravesh, P.E.: Newtonian-based methodologies in multi-body dynamics. Proceedings of the Institution of Mechanical Engineers, Part K: Journal of Multi-body Dynamics, 222, 277-288 (2008)

15. Routh, E.T.: Dynamics of a System of Rigid Bodies. Macmillan, London (1905)

16. Orlandea, N., Chace, M.A., Calahan, D.A.: A sparsity oriented approach to the dynamic analysis and design of mechanical systems - parts 1 and 2. Journal of Engineering for Industry, 99, 773-784 (1977)

17. Kane, T.R., Levinson, D.A.: Dynamics: Theory and Applications. McGraw-Hill, New York (1985)

18. Greenwood, D.T.: Principles of Dynamics. $2^{\text {nd }}$ ed. Englewood Cliffs, Prentice-Hall, New Jersey (1988)

19. Huston, R.L.: Multibody Dynamics - Modeling and Analysis Methods. Applied Mechanics Review, 44(3), 109-117 (1991) 
20. Pfeifer, F., Glocker, C.: Multibody Dynamics with Unilateral Constraints. Wiley, New York (1996)

21. Nikravesh, P.E.: Planar Multibody Dynamics: Formulation, Programming, and Applications, CCR Press, London (2008)

22. Guo, W., Wang, T.: A methodology for simulations of multi-rigid body systems with topology changes. Multibody System Dynamics, 32(1), 25-38 (2015)

23. Nikravesh, P.E.: Some methods for dynamic analysis of constrained mechanical systems: A survey. Computer-Aided analysis and Optimization of Mechanical System Dynamics, edited by E.J. Haug, Springer Verlag, Berlin, Germany, 351-368 (1984)

24. Rosen, A., Edelstein, E.: Investigation of a new formulation of the Lagrange method for constrained dynamic systems. Journal of Applied Mechanics, 64, 116-122 (1997)

25. Eich-Soellner, E., Führer, C.: Numerical Methods in Multibody Dynamics, vol. 45. Springer, Berlin (1998)

26. Von Schwerin, R.: Multibody System Simulation: Numerical Methods, Algorithms, and Software, vol. 7. Springer, Berlin (1999)

27. Blajer, W.: Elimination of constraint violation and accuracy aspects in numerical simulation of multibody systems. Multibody System Dynamics, 7, 265-284 (2002)

28. Laulusa, A., Bauchau, O.A.: Review of classical approaches for constraint enforcement in multibody systems. J. Comput. Nonlinear Dyn. 3(1), 011004 (2007)

29. Bauchau, O.A., Laulusa, A.: Review of contemporary approaches for constraint enforcement in multibody systems. J. Comput. Nonlinear Dyn. 3(1), 011005 (2007)

30. Masarati, P.: Constraint stabilization of mechanical systems in ordinary differential equations form. Proceedings of the Institution of Mechanical Engineers, Part K: Journal of Multi-body Dynamics, 225, 12-33 (2011)

31. Flores, P., Machado, M., Seabra, E., Silva, M.T., A parametric study on the Baumgarte stabilization method for forward dynamics of constrained multibody systems. Journal of Computational and Nonlinear Dynamics, 6(1), 0110191-9 (2011)

32. Neto, M.A., Ambrósio, J.: Stabilization methods for the integration of DAE in the presence of redundant constraints. Multibody System Dynamics, 10, 81-105, (2003)

33. Fisette, P., Vaneghem, B.: Numerical integration of multibody system dynamic equations using the coordinate partitioning method in an implicit Newmark scheme. Computer Methods in Applied Mechanics and Engineering, 135, 85-105 (1996)

34. Ascher, U., Chin, H., Petzold, L., Reich, S.: Stabilization of constrained mechanical systems with DAEs and invariant manifolds. Mech. Struct. Mach. 23, 135-157 (1995)

35. Cuadrado, J., Cardenal, J., Bayo, E.: Modeling and solution methods for efficient real-time simulation of multibody dynamics. Multibody System Dynamics, 1(3), 259-280 (1997)

36. Schwerin, R.: Multibody System Simulation. Numerical Methods, Algorithms and Software. Springer, Berlin (1999)

37. Yu, Q., Chen, I.M.: A direct violation correction method in numerical simulation of constrained multibody systems. Comput. Mech. 26(1), 52-57 (2000)

38. Aghili, F., Piedbœuf, J.-C.: Simulation of motion of constrained multibody systems based on projection operator. Multibody System Dynamics, 10(1), 3-16 (2003)

39. Udwadia, F.E., Phohomsiri, P.: Explicit equations of motion for constrained mechanical systems with singular mass matrices and applications to multi-body dynamics. Proc. R. Soc. A, Math. Phys. Eng. Sci., 462(2071), 2097-2117 (2006)

40. Flores, P., Pereira, R., Machado, M., Seabra, E.: Investigation on the Baumgarte Stabilization Method for Dynamic Analysis of Constrained Multibody Systems. Edited by: Ceccarelli, M., Proceedings of the EUCOMES 08, The Second European Conference on Mechanism Science, Cassino, Italy, September 17-20, 2008, pp. 305-312 (2009)

41. Vlasenko, D., Kasper, R.: Implementation of consequent stabilization method for simulation of multibodies described in absolute coordinates. Multibody System Dynamics, 22, 297-319 (2009)

42. Mariti, L., Belfiore, N.P., Pennestrì, E., Valentini, P.P.: Comparison of solution strategies for multibody dynamics equations. Int. J. Numer. Methods Eng. 88(7), 637-656 (2011)

43. Müller, A.: Motion equations in redundant coordinates with application to inverse dynamics of constrained mechanical systems. Nonlinear Dynamics, 67, 2527-2541 (2012)

44. Uchida, T., Vyasarayani, C.P., Smart, M., McPhee, J.: Parameter identification for multibody systems expressed in differential-algebraic form. Multibody System Dynamics, 31(4), 393-403 (2014) 
45. Baumgarte, J.: Stabilization of constraints and integrals of motion in dynamical systems. Computer Methods in Applied Mechanics and Engineering, 1, 1-16 (1972)

46. Baumgarte, J.: A New Method of Stabilization for Holonomic Constraints. Journal of Applied Mechanics, 50, 869-870 (1983)

47. Chang, C.O.: Dynamic Analysis and Optimum Design of Mechanical Systems with Constraint Violation Stabilization Method. Ph.D. Dissertation, Department of Mechanical Engineering, University of Iowa (1984)

48. Barzel, R., Barr, A.: A modeling system based on dynamic constraints. Computer Graphics, 22(4), 179-188 (1988)

49. Platt, J.C., Barr, A.: Constraint methods for flexible models. Computer Graphics, 22(4), 279-288 (1988)

50. Yoon, S.: Real-Time Simulation of Constrained Dynamic Systems. Ph.D. Dissertation, Department of Aerospace Engineering, University of Michigan, Ann Arbor, MI (1990)

51. Ostermeyer, G.P.: On Baumgarte stabilization for differential algebraic equations, in: E.J. Haug, R.C. Deyo (Eds.), Real-Time Integration Methods for Mechanical System Simulations, NATO ASI Series, Vol. F69, Springer, Berlin, 193-207 (1990)

52. Chin, H.S.: Stabilization Methods for Simulations of Constrained Multibody Dynamics. Ph.D. Dissertation, Department of Mathematics, The University of British Columbia, Canada (1995)

53. Eich, E., Hanke, M.: Regularization methods for constrained mechanical multibody systems. Zeitschrift fur angewandte Mathematik und Mechanik, 75(10), 761-773 (1995)

54. Baraff, D.: Linear-time dynamics using Lagrange multipliers. Proceedings of SIGGRAPH 1996, ACM Press/ACM SIGGRAPH, Computer Graphics Proceedings, 137-146 (1996)

55. Lin, S.T., Hong, M-C.: Stabilization method for numerical integration of multibody mechanical systems. Journal Mechanical Design, 120, 565-572 (1998)

56. Chiou, J.C., Wu, S.D.: Constraint violation stabilization using input-output feedback linearization in multibody dynamic analysis. Journal of Guidance, Control, and Dynamics, 21(2), 222-228 (1998)

57. Junkins, J.L., Akella, M.R., Kurdila, A.J.: Adaptive realization of desired constraint stabilization dynamics in the control of multibody systems. Philosophical Transactions: Mathematical, Physical \& Engineering Sciences, 359(1788), 2231-2249 (2001)

58. Hajzman, M., Polach, P.: Application of stabilization techniques in the dynamic analysis of multibody systems. Applied and Computational Mechanics, 1, 479-488 (2007)

59. Machado, M., Costa, J., Seabra, E., Flores, P.: The effect of the lubricated revolute joint parameters and hydrodynamic force models on the dynamic response of planar multibody systems. Nonlinear Dynamics, 69(1-2), 635-654 (2012)

60. Ascher, U.R., Chin H., Reich, S.: Stabilization of DAEs and invariant manifolds. Numerische Methematik, 67(2), 131-149 (1994)

61. Chang, C.O., Nikravesh, P.E.: An adaptive constraint violation stabilization method for dynamic analysis of mechanical systems. Journal of Mechanisms, Transmissions, and Automation in Design, 107, 488-492 (1985)

62. Bae, D.S., Yang, S.M.: A stabilization method for kinematic and kinetic constraint equations. In E.J. Haug and R.C. Deyo, editors, Real-Time Integration Methods for Mechanical System Simulation, 209-232. Springer-Verlag, Berlin, Heidelberg, 1990.

63. Yoon, S., Howe, R.M., Greenwood, D.T.: Stability and accuracy analysis of Baumgarte's constraint violation stabilization method. Journal of Mechanical Design, 117, 446-453 (1995)

64. Lin, S.T., Hong, M.C.: Stabilization method for numerical integration of multibody mechanical systems. Journal of Mechanical Design, 120, 565-572 (1998)

65. Lin, S-T., Huang, J-N.: Stabilization of Baumgarte's method using the Runge-Kutta approach. Journal of Mechanical Design, 124, 633-641 (2002)

66. Park, K.C., Chiou, J.C.: Stabilization of computational procedures for constrained dynamical systems. Journal of Guidance, Control, and Dynamics, 11, 365-370 (1988)

67. Park, K.C., Chiou, J.C., Downer, J.D.: Explicit-implicit staggered procedure for multibody dynamics analysis. Journal of Guidance, Control, and Dynamics, 13(3), 562-570 (1990)

68. Ostermeyer, G.P.: On Baumgarte stabilization for differential algebraic equations. In E.J. Haug and R.C. Deyo, editors, Real-Time Integration Methods for Mechanical System Simulation, 193-207. Springer-Verlag, Berlin, Heidelberg (1990)

69. Yoon, S., Howe, R.M., Greenwood, D.T.: Constraint Violation Stabilization Using Gradient Feedback in Constrained Dynamics Simulation. Journal of Guidance, Control, and Dynamics, 15(6), 1467-1474 (1992) 
70. Hong, M., Choi, M-H., Jung, S., Welch, S., Trapp, J.: Effective Constrained Dynamic Simulation Using Implicit Constraint Enforcement. Proceedings of the 2005 IEEE International Conference on Robotics and Automation, Barcelona, Spain, pp. 4531-4536 (2005)

71. Hong, M., Welch, S., Trapp, J., Choi, M.H.: Implicit Constraint Enforcement for Rigid Body Dynamic Simulation. Computational Science - ICCS 2006, Lecture Notes in Computer Science, 3991, 490-497 (2006)

72. Weijia, Z., Zhenkuan, P., Yibing, W.: An automatic constraint violation stabilization method for differential/algebraic equations on multibody system dynamics. Applied Mathematics and Mechanics, 21(1), 103-108 (2000)

73. Cline, M.B., Pai, D.K.: Post-Stabilization for Rigid Body Simulation with Contact and Constraints. In Proceedings of the IEEE International Conference on Robotics and Automation, 8p. (2003)

74. Bayo, E., Jalón, J.G., Serna, M.A.: A modified Lagrangian formulation for the dynamic analysis of constrained mechanical systems. Comput. Methods Appl. Math. 71(2), 183-195 (1988)

75. Bayo, E., Avello, A.: Singularity-free augmented Lagrangian algorithms for constrained multibody dynamics. Nonlinear Dynamics, 5(2), 209-231 (1994)

76. Terze, Z., Lefeber, D., Muftic, O.: Null Space Integration Method for Constrained Multibody Systems with No Constraint Violation. Multibody System Dynamics, 6, 229-243 (2001)

77. Braun, D.J., Goldfarb, M.: Eliminating constraint drift in the numerical simulation of constrained dynamical systems. Comput. Methods Appl. Math. 198(37-40), 3151-3160 (2009)

78. Blajer, W.: Methods for constraint violation suppression in the numerical simulation of constrained multibody systems - a comparative study. Comput. Methods Appl. Math. 200(13-16), 1568-1576 (2011)

79. Wehage, R.A., Haug, E.J.: Generalized coordinate partitioning for dimension reduction in analysis of constrained systems. Journal of Mechanical Design, 104, 247-255 (1982)

80. Nikravesh, P.E., Haug, E.J.: Generalized Coordinate Partitioning for Analysis of Mechanical System with Nonholonomic Constraints. Journal of Mechanisms, Transmissions, and Automation in Design, 105(3), 379384 (1983)

81. Haug, E.J., Yen, J.: Generalized coordinate partitioning methods for numerical integration of differentialalgebraic equations of dynamics. In Real-time Integration Methods for Mechanical System Simulation, Haug. E.J., Deyo. R.C. (eds). NATO ASI Series, Vol. 69. Springer, Berlin, 97-114 (1991)

82. Arabyan, A., Wu, F.: An improved formulation for constrained mechanical systems. Multibody Systems Dynamics, 2(1), 49-69 (1998)

83. Terze, Z., Naudet, J.: Geometric properties of projective constraint violation stabilization method for generally constrained multibody systems on manifolds. Multibody Systems Dynamics, 20, 85-106 (2008)

84. Terze, Z., Naudet, J.: Structure of optimized generalized coordinates partitioned vectors for holonomic and non-holonomic systems. Multibody Systems Dynamics, 24(2), 203-218 (2010)

85. Blajer, W., Schiehlen, W., Schirm, W.: A projective criterion to the coordinate partitioning method for multibody dynamics. Arch. Appl. Mech. 64, 86-98 (1994)

86. Nikravesh, P.E., Skinivasan, M.: Generalized co-ordinate partitioning in static equilibrium analysis of largescale mechanical systems. Int. J. Numer. Methods Eng. 21, 451-464 (1985)

87. Jalón, J.G., Unda, J., Avello, A., Jiménez, J.M.: Dynamic analysis of three-dimensional mechanisms in natural coordinates. Computer Methods in Applied Mechanics and Engineering, 56, 309-327 (1986)

88. Ider, S.K., Amirouche, E.M.L.: Coordinate reduction in constrained spatial dynamic systems - a new approach. Journal of Applied Mechanics, 55, 899-905 (1988)

89. Fuhrer, C., Schwertassek, R.: Generation and solution of multibody systems equations. International Journal Non-Linear Mechanics, 25, 127-141 (1990)

90. Jalón, J.G., Gutiérrez-López, M.D.: Multibody dynamics with redundant constraints and singular mass matrix: existence, uniqueness, and determination of solutions for accelerations and constraint forces. Multibody System Dynamics, 30(3), 311-341 (2013)

91. Wehage, K.T., Wehage, R.A., Ravani, B.: Generalized coordinate partitioning for complex mechanisms based on kinematic substructuring. Mechanism and Machine Theory, 92, 464-483 (2015)

92. Carpinelli, M., Gubitosa, M., Mundo, D., Desmet, W.: Automated independent coordinates' switching for the solution of stiff DAEs with the linearly implicit Euler method. Multibody System Dynamics, 36(1), 67-85 (2016)

93. Lubich, C.: Extrapolation integrators for constrained multibody systems. Impact Comput. Sci. Eng. 3(3), 213-234 (1991) 
94. Andrzejewski, T., Bock, H.: Recent advances in the numerical integration of multibody systems. In: Schiehlen, W. (ed.) Advanced Multibody System Dynamics. Solid Mechanics and Its Applications, vol. 20, pp. 127-151. Springer, The Netherlands (1993)

95. Yu, Q., Chen, I.M.: A direct violation correction method in numerical simulation of constrained multibody systems. Computational Mechanics, 26(1), 52-57 (2000).

96. Eich, E.: Convergence results for a coordinate projection method applied to mechanical systems with algebraic constraints. SIAM J. Numer. Anal. 30(5), 1467-1482 (1993)

97. Yoon, S., Howe, R.M., Greenwood, D.T.: Geometric elimination of constraint violations in numerical simulation of Lagrangian equations. Journal of Mechanical Design, 116, 1058-1064 (1994)

98. Blajer, W.: An orthonormal tangent space method for constrained multibody systems. Computer Methods in Applied Mechanics and Engineering, 121, 45-57 (1995)

99. Blajer, W.: A geometric unification of constrained system dynamics. Multibody Syst. Dyn. 1(1), 3-21 (1997).

100. Aghili, F., Piedboeuf, J.C.: Simulation of motion of constrained multibody systems based on projection operator. Multibody System Dynamics, 10(1), 3-16 (2003).

101. Tseng, F.-C., Ma, Z-D., Hulbert, G.M.: Efficient numerical solution of constrained multibody dynamics systems. Computer Methods in Applied Mechanics and Engineering, 192, 439-472 (2003)

102. Nikravesh, P.E.: Initial condition correction in multibody dynamics. Multibody System Dynamics, 18:107115 (2007)

103. Zhang, J., Liu, D., Liu, Y.: A constraint violation suppressing formulation for spatial multibody dynamics with singular mass matrix. Multibody System Dynamics, 36(1), 87-110 (2016)

104. Bayo, E., Ledesma, R.: Augmented Lagrangian and mass-orthogonal projection methods for constrained multibody dynamics. Nonlinear Dynamics, 9(1-2), 113-130 (1996)

105. Orden, J.G.: Energy considerations for the stabilization of constrained mechanical systems with velocity projection. Nonlinear Dynamics, 60(1-2), 49-62 (2010)

106. Orden, J.G., Conde Martín, S.: Controllable velocity projection for constraint stabilization in multibody dynamics. Nonlinear Dyn. 68(1-2), 245-257 (2012)

107. Terze, Z., Müller, A. Zlatar, D.: Lie-group integration method for constrained multibody systems in state space. Multibody System Dynamics, 34, 275-305 (2015)

108. Müller, A., Terze, Z.: The significance of the configuration space Lie group for the constraint satisfaction in numerical time integration of multibody systems. Mechanism and Machine Theory, 82, 173-202 (2014)

109. Tian, Q., Xiao, Q., Sun, Y., Hu, H., Liu, H., Flores, P.: Coupling dynamics of a geared multibody system supported by ElastoHydroDynamic lubricated cylindrical joints. Multibody System Dynamics, 33(3), 259284 (2015)

110. Qi, Z., Wang, G., Zhang, Z.: Contact analysis of deep groove ball bearings in multibody systems. Multibody System Dynamics, 33(2), 115-141 (2015)

111. Blajer, W.: Augmented Lagrangian formulation: geometrical interpretation and application to systems with singularities and redundancy. Multibody System Dynamics 8(2), 141-159 (2002).

112. Haghshenas-Jaryani, M., Bowling, A.: A new switching strategy for addressing Euler parameters in dynamic modeling and simulation of rigid multibody systems. Multibody System Dynamics 30(2), 185-197 (2013)

113. Wojtyra, M., Fraczek, J.: Comparison of selected methods of handling redundant constraints in multibody system simulations. Journal of Computational and Nonlinear Dynamics, 8, 021007 (2013)

114. Unda, J., Jalón, J.G., Losantos, F., Emparantza, R.: A comparative study of different formulations of the dynamic equations of constrained mechanical systems. Journal of Mechanisms, Transmissions, and Automation in Design, 109, 466-474 (1987)

115. Udwadia, F.E., Kalaba, R.E.: A new perspective on constrained motion. Proc. R. Soc. Lond.,Math. Phys. Sci. 439(1906), 407-410 (1992)

116. Erberhard, P., Schielen, W.: Computational dynamics of multibody systems: history, formalisms, and applications. Journal of Computational and Nonlinear Dynamics, 1, 3-12 (2006)

117. Ruzzeh, B., Kövecses, J.: A penalty formulation for dynamic analysis of redundant mechanical systems. Journal of Computational and Nonlinear Dynamics, 6, 021008 (2011)

118. González, F., Kövecses, J.: Use of penalty formulations in the dynamic simulation and analysis of redundantly constrained multibody systems. Multibody System Dynamics, 29(1), 57-76 (2013)

119. Kim, J.K., Chung, I.S., Lee, B.H.: Determination of the Feedback Coefficients for the Constraint Violation Stabilization Method. Proceedings of the Institution of Mechanical Engineers, Part C: Journal of Mechanical Engineering Science, 204, 233-242 (1990) 
120. Angles, J., Lee, S.: The formulation of dynamical equations of holonomic mechanical systems using a natural orthogonal complement. Journal of Applied Mechanics, 55, 243-244 (1988)

121. Agrawal, O.P., Saigal, S.: Dynamic analysis of multi-body systems using tangent coordinates. Computers \& Structures, 31(3), 349-355 (1989)

122. Shabana, A.A.: Computational Dynamics. Wiley, New York (1994)

123. Jalón, J.G., Callejo, A., Hidalgo, A.F.: Efficient Solution of Maggi’s Equations. Journal of Computational and Nonlinear Dynamics, 7(2), 021003 (2012)

124. Serban, R., Haug, E.J.: Globally Independent Coordinates for Real-Time Vehicle Simulation. Journal of Mechanical Design, 122(4), 575-582 (2000)

125. Malczyk, P., Frączek, J.: A divide and conquer algorithm for constrained multibody system dynamics based on augmented Lagrangian method with projections-based error correction. Nonlinear Dynamics, 70(1), 871889, (2012)

126. Bayo, E., Jalón, J.G., Avello, A., Cuadrado, J.: An efficient computational method for real time multibody dynamic simulation in fully cartesian coordinates. Computer Methods in Applied Mechanics and Engineering, 92(3), 377-395 (1991)

127. Flores, P., Nikravesh, P.E.: Comparison of different methods to control constraints violation in forward multibody dynamics. Proceedings of the ASME Design Engineering Technical Conference, Volume 7 A. ASME 2013 International Design Engineering Technical Conferences and Computers and Information in Engineering Conference, IDETC/CIE 2013, Portland, OR, United States; 4-7 August, Code 103368 (2013)

128. Cuadrado, J., Cardenal, J., Morer, P., Bayo, E.: Intelligent simulation of multibody dynamics: Space-state and descriptor methods in sequential and parallel computing environments. Multibody System Dynamics, 4(1), 55-73, (2000)

129. Dopico, D., González, F., Cuadrado, J., Kövecses, J.: Determination of Holonomic and Nonholonomic Constraint Reactions in an Index-3 Augmented Lagrangian Formulation With Velocity and Acceleration Projections. Journal of Computational and Nonlinear Dynamics, 9(4), 041006, 9 pages (2014).

130. González, F., Dopico, D., Pastorino, R., Cuadrado, J.: Benchmarking of augmented Lagrangian and Hamiltonian formulations for multibody system dynamics. Proceedings of the ECCOMAS Thematic Conference on Multibody Dynamics June 29 - July 2, 2015, Barcelona, Catalonia, Spain, 1548-1559 (2015)

131. González, F., Dopico, D., Pastorino, R., Cuadrado, J.: Behaviour of augmented Lagrangian and Hamiltonian methods for multibody dynamics in the proximity of singular configurations. Nonlinear Dynamics, DOI: 10.1007/s11071-016-2774-5 (2016)

132. Flores, P.: A methodology for quantifying the position errors due to manufacturing and assemble tolerances. Journal of Mechanical Engineering, 57(6), 457-467 (2011)

133. Moore, E.H.: On the reciprocal of the general algebraic matrix. Bulletin of the American Mathematical Society, 26(9), 394-395 (1920)

134. Penrose, R.: A generalized inverse for matrices. Proceedings of the Cambridge Philosophical Society, 51, 406-413 (1955).

135. Rao, C.R., Mitra, S.K.: Generalized Inverse of Matrices and its Applications. John Wiley and Sons, New York (1971)

136. Mani, N.K., Haug, E.J.: Atkinson KE. Singular value decomposition for analysis of mechanical system dynamics. Journal of Mechanisms, Transmissions, and Automation in Design, 107, 82-87 (1985)

137. Singh, R.P., Likins. P.W.: Singular value decomposition for constrained mechanical systems. Journal of Applied Mechanics, 52, 943-948 (1985)

138. Kim, S.S., Vanderploeg, M.J.: QR decomposition for state space representation of constrained mechanical dynamical systems. Journal of Mechanisms, Transmissions, and Automation in Design, 108, 183-188, (1986)

139. Meijaard, J.P.: Applications of the single value decomposition in dynamics. Computer Methods in Applied Mechanics and Engineering, 103, 161-173 (1993)

140. McPhee, J., Shi, P., Piedbuf, J.-C.: Dynamics of Multibody Systems Using Virtual Work and Symbolic Programming. Mathematical and Computer Modelling of Dynamical Systems: Methods, Tools and Applications in Engineering and Related Sciences, 8(2), 137-155 (2002)

141. Iltis Data Package, IAVSD Workshop, Herbertov, Czechoslovakia, September 1990. 